\title{
Accurate specification of water availability shows its importance for global crop production
}

\author{
Jonathan Proctor ${ }^{1}$, Angela Rigden ${ }^{2}$, Duo Chan ${ }^{3}$, and Peter Huybers ${ }^{2}$ \\ ${ }^{1}$ Center for the Environment and Data Science Initiative, Harvard University, Cambridge, MA \\ ${ }^{2}$ Department of Earth and Planetary Sciences, Harvard University, Cambridge, MA \\ ${ }^{3}$ Department of Physical Oceanography, Woods Hole Oceanographic Institution, Woods Hole, \\ MA
}

We welcome your feedback on this research; feel free to contact the authors via email (jproctor1@fas.harvard.edu, angelarigden@fas.harvard.edu, duochan@g.harvard.edu, phuybers@fas.harvard.edu). This is a non-peer-reviewed pre-print. Subsequent revisions of this manuscript may include revisions based on feedback and the peer-review process. If accepted in a peer-reviewed journal, the final version of this manuscript will be available via the "Peerreviewed Publication DOI" link on the EarthArXiv page for this paper. 


\title{
Accurate specification of water availability shows its importance for global crop production
}

\author{
Jonathan Proctor ${ }^{1 *}$, Angela Rigden ${ }^{2}$, Duo $\mathrm{Chan}^{3}$, and Peter Huybers ${ }^{2}$ \\ ${ }^{1}$ Center for the Environment and Data Science Initiative, Harvard University, Cambridge, MA \\ ${ }^{2}$ Department of Earth and Planetary Sciences, Harvard University, Cambridge, MA \\ ${ }^{3}$ Department of Physical Oceanography, Woods Hole Oceanographic Institution, Woods Hole, MA \\ *To whom correspondence should be addressed: jproctor1@fas.harvard.edu
}

November 2021

\begin{abstract}
It is well established that warming temperatures damage the yields of many crops across the globe. Yet the influence of water supply on global agricultural yield and its relation to water demand and direct temperature stress is unclear. A number of global studies found a minor influence for precipitation, whereas some regional analyses suggest a more prominent role for water availability. Here, we use satellite-based measurements to quantify how soil moisture and temperature jointly influence the global productivity of maize, soybeans, millet, and sorghum. Relative to empirical models using precipitation as a proxy for water availability, models using soil moisture better separate water supply stress from correlated heat stress, leading to a 30 to $120 \%$ increase in explained variance of inter-annual yield anomalies across crops. Historic yield anomalies are equally determined by temperature and soil moisture, whereas projected damages associated with climate change are substantially larger for temperature. Globally, yield damages of $-9 \%$ to $-32 \%$ are predicted across crops under SSP $5-8.5$ between 2015-2035 and 2080-2100. Projections using temperature and precipitation, instead of soil moisture, overestimate the magnitude of damages to agricultural productivity because they confound heat stress and dryness stress, and because dryness associated with historically hot days is proportionately more severe than that expected for global warming. These findings indicate that use of remotely sensed measurements of soil moisture improve the representation of water supply in empirical crop models and document the importance of accurately measuring and modelling the influence of water supply to predict historic and future changes in global agricultural productivity.
\end{abstract}




\section{Introduction}

Agricultural productivity is a key determinant of global food security and well-being. It is well understood that warming has and will increasingly cause reductions in global crop yields $[1,2,3,4]$. Crop water supply measured using precipitation, however, was found to have only a minor influence on yield at global $[5,6,4,7]$ and regional $[1,3]$ scales. Recently, regional studies of U.S. agriculture have, instead, found a substantial influence of soil moisture [8, 9]. Although it is obvious that water is necessary for agriculture, both the quantitative influence of water supply on the yields of different crops, and how the influence of water supply relates to that of water demand and direct temperature stress remain poorly understood at the global scale.

Crop water supply is influenced by root structure and root-zone soil moisture, which depends upon a multitude of factors including rainfall, evaporation, runoff, soil properties and agricultural practices. Limited water supply damages yields by encouraging root growth at the cost of yield and by slowing photosynthesis [10]. Under low water supply, plants may close their stomata in order to conserve water, but thereby reduce carbon uptake, photosynthetic rates, and yield [11]. In severe cases low soil moisture can lead to cavitation, embolism and crop mortality [12]. Water demand is primarily influenced by vapor-pressure deficit (VPD), which controls the rate of evaporation and transpiration. Under high VPD, plants may similarly close their stomata in order to conserve water at the cost of carbon uptake [11]. In addition to influencing water demand through VPD, high temperatures can directly reduce yields by damaging plant structures, enzymes and biological membranes, disrupting reproductive development, shortening grain filling, and reducing net photosynthesis rates $[13,14]$. Low temperatures can also reduce yields by slowing growth rates, reducing photosynthetic efficiency, and negatively affecting morphology, and physiology [13].

Quantifying the influences of water supply, water demand and direct temperature stress on agricultural productivity is necessary to skillfully predict environmentally-induced yield anomalies, and to direct adaptive investments such as heat-tolerant or drought-resistant varietals. Imprecisely accounting for water supply or temperature effects is liable to bias estimates because these variables strongly covary [15]. For example, dry soil is often associated with high temperature and high vapor pressure deficit, especially at synoptic timescales [16]. It follows that mismeasurement of water supply can lead to both inflated estimates of the damages from temperature stress and under-estimation of the temperature at which warming switches from being beneficial to damaging [9]. Such mismeasurement can also lead to an underestimation of the relative influence of water supply on yield, compared with that of water demand and direct temperature stress. Although modeling the influence of temperature stress without directly accounting for water supply might capture both correlated and induced water supply stress, the potential for climate trends to be distinct from the synoptic covariance among these variables makes it important to accurately represent the effects of both heat and water.

Water supply is commonly represented in statistical crop models using measurements of precipitation. Precipitation is generally found to benefit yields, excepting in cases of oversupply, but is estimated to have only a minor effect on yield relative to temperature $[3,4,5,6,7]$, sometimes leading to its omission from main lines of analyses all together $[1,17]$. Such a minor role may reflect that precipitation is a poor proxy for crop water supply due to heterogeneous evaporation, infiltration, and runoff across space and time [1]. Furthermore, 
in these models temperature may represent not only direct effects but also assume the role of representing changes in water demand (through changes in VPD) and water supply (both through changes in VPD which influence evapotranspiration and because changes in water supply influence temperature through latent cooling) [14, 18, 19, 20].

Analyses have separated the effects of temperature stress from water supply stress by analyzing irrigated areas [21, 22]. Such models, however, do not provide estimates of the influence of water supply itself, which limits the ability to project effects of potential future changes in water supply on yield. Analyses that leverage measurements of surface soil moisture and VPD to separate the effects of water supply and water demand on the productivity of global unmanaged ecosystems suggest that both can have substantial effects $[23,24]$, though environmental influences on crop yields may differ from those on unmanaged systems due to agricultural management practices and differences between biomass growth and edible yield [25]. Recent empirical studies of agriculture in the United States suggest that measurements of soil moisture can capture the influence of water supply on crop yield better than precipitation, leading to a better model fit $[8,9]$ and enabling better separation of the influences of water supply and water demand on yield.

Understanding how the global yields of a range of staple crops respond to changes in water and temperature stress is critical for understanding the potential influence of climate change on food security. Here, we analyze how root-zone soil moisture and near-surface atmospheric temperature combine to influence the yields of four staple crops globally. In this formulation, soil moisture represents water supply and temperature represents both water demand and direct heat effects. We use satellite-based soil moisture observations (CCI SM v06.1) [26, 27], station measurements of precipitation and temperature [28], and national records of crop yield from the Food and Agriculture Organization of the United Nations [29] (Extended Data Fig. 4) covering 2007-2018 (Methods).

The joint influence of either temperature and soil moisture (TS) or temperature and precipitation (TP) are estimated using panel regression models that flexibly estimate the influence of TS or TP on yields [30]. Our models account for time-constant differences across space, such as soil type, by including an intercept for each location, and for time-trending factors within each country, such as income and technological improvements, using a linear time trend. We evaluate the model using cross-validation performance (Extended Data Table 1, Methods) and by checking that estimated responses of crop yield to temperature, soil moisture, and precipitation are consistent with physiological expectations and accord with previous studies $[3,6,9]$ (Fig. 2). TS models are shown to better capture the influence of water supply than TP models, and the differences between these models for future agricultural productivity are illustrated by applying them to daily output from six earth system models from the Coupled Model Intercomparison Project phase 6 (CMIP6) [31] (Methods).

\section{Results}

Soil moisture improves representation of crop water supply in empirical crop models. The TS model recovers temperature and soil moisture influences that are consistent with physiological understanding. Across crops, and in line with previous analyses $[1,4,6,32]$, yields have a concave response to temperature stress, with warming benefiting yields up to a critical temperature, after which higher temperatures damage yield (Fig. 2, 
maize $\mathrm{p}<0.01$, soybeans $\mathrm{p}<0.01$, sorghum $\mathrm{p}=0.03$, and millet $\mathrm{p}=0.09$ relative to a null of no effect). Crops also generally show an increase in yields with increasing soil moisture up to a point around $0.3 \frac{\mathrm{cm}^{3}}{\mathrm{~cm}^{3}}$, after which increased soil moisture has little effect on yield (maize $\mathrm{p}<0.01$, soybeans $\mathrm{p}<0.01$, sorghum $\mathrm{p}<0.01$ and millet $\mathrm{p}=0.12$ ). The nonlinear relationship between soil moisture and temperature anomalies in croplands has a similar structure whereby increased soil moisture is associated with lowered temperature, up until soil moisture values of $0.3 \frac{\mathrm{cm}^{3}}{\mathrm{~cm}^{3}}$, after which temperature anomalies no longer depend on soil moisture (Extended Data Fig. 2D), illustrating the relationship between soil moisture, photosynthesis, evapotranspiration, and resulting latent cooling.

The TS model captures the vast majority of global variability in crop yields (in-sample model adjusted $\mathrm{R}^{2}$ values of 0.97 for maize, 0.93 for soybeans, 0.94 for sorghum, and 0.92 for millet). The variations explained by temperature and soil moisture after country means and trends are removed (i.e., out-of-sample within $\mathrm{R}^{2}$ values of 0.095 for maize, 0.129 for soybeans, 0.044 for sorghum, and 0.031 for millet) are competitive with previous global analyses of climate influences on national crop yields, though direct comparison is complicated by differences in methodologies [4, 6, 32].

The TS model better captures water supply stress than the TP model (Extended Data Tab. 1). Model fit measured by cross-validation within $\mathrm{R}^{2}$ increases for all crops (47\% for maize, $31 \%$ for soybeans, $116 \%$ for sorghum and $28 \%$ for millet). The increase in variance explained is statistically significant for maize $(\mathrm{p}<0.01)$ and soybeans $(\mathrm{p}<0.01)$, but marginal for sorghum $(\mathrm{p}=0.06)$ and insignificant for millet $(\mathrm{p}=0.64)$ (Supplementary Table 1). Including precipitation in addition to soil moisture and temperature does not further improve performance, indicating that soil moisture fully captures the influence of precipitation on water supply.

\section{Improved representation of water supply enables separation of water supply} stress and temperature stress. We find that water supply and temperature stress contribute approximately equally to climate-induced historical global yield variability. In models of yield as a nonlinear function of a single environmental variable, soil moisture explains more of the variability in yield than does temperature for maize ( $72 \%$ for soil moisture versus $65 \%$ for temperature) and sorghum (88\% versus 40\%), and less of the variability for soybeans (69\% versus $80 \%)$ and millet (73\% versus $95 \%)$. Variability explained is reported as the fraction relative to the full TS model (Extended Data Table 1). In contrast, precipitation alone captures only $22 \%$ of maize, $16 \%$ of sorghum, $28 \%$ of soybeans, and $8 \%$ of millet variations relative to the variance explained by the TS model.

It follows that temperature and soil moisture also contribute relatively equally to yield anomalies predicted by the TS model over our sample - soil moisture contributes more to the predicted variability in yield anomalies for maize ( $48 \%$ for soil moisture versus $38 \%$ for temperature, and $14 \%$ for their covariance), soybeans (44\% versus $35 \%$, and $22 \%$ ), and sorghum (84\% versus $26 \%$, and $-10 \%$ ), and less to the variability for millet (30\% versus $52 \%$, and 19\%). Contribution to yield anomalies is reported as the variance of yield anomalies due to the temperature or soil moisture component of the TS model as well as their covariance, relative to the the variance of yield anomalies predicted by the full TS model. The relatively even contribution of temperature and soil moisture components of the TS model to predic- 
tions of historical yield anomalies is illustrated in a reconstruction of historical variations around the 2012 drought and heat wave in the United States (Extended Data Fig. 5).

These findings are consistent when using different model specifications and cross-validation approaches (Extended Data Table 1, Supplementary Information Discussion 2, Supplementary Information Tables 1 and 2). The finding that water supply stress and temperature stress contribute equally to historic global agricultural productivity variability is consistent with previous studies of natural vegetation productivity that find that soil moisture and temperature stress can both have substantial effects [23, 24].

\section{Separation of water supply stress and temperature stress in the TS model re-} moves damages due to dry soils from the estimated influence of extreme heat. Beyond the fact that temperature and soil moisture both independently influence yield, the importance of accounting for both is highlighted by their anticorrelation [15]. Daily growingseason temperature and soil moisture across global croplands have a Pearson's correlation coefficient of $\rho_{T S}=-0.19$ (Fig. 1, Extended Data Fig. 3), and this anticorrelation grows to $\alpha_{S P}=-0.30$ for detrended national growing-season average conditions (Extended Data Fig. 2). Anticorrelation is expected both because increased soil moisture drives cooling through evapotranspiration and increased temperature drives drying, also through evapotranspiration.

Although studies commonly proxy for water supply using precipitation, the correlation between growing-season precipitation and soil moisture is weak at the daily, grid-scale level $\left(\rho_{S P}=0.22\right.$, Fig 1$)$ and remains imperfect at the growing-season average, country level $\left(\alpha_{S P}=0.74\right.$, Extended Data Figs. 2,3). The disconnect between precipitation and soilmoisture at the daily, grid-scale level is consistent with the reduced performance of TP models.

Correlation between temperature and soil moisture, along with precipitation being a poor proxy for soil moisture, leads to temperature in the TP model capturing not only the influence of temperature stress, but also water supply stress. Part of this water supply stress associated with temperature stress in the TP model is due to temperature-driven increases in VPD and evapotranspiration, and thus can be considered part of the total effect of a change in temperature. However, another part of the associated water supply stress is due to soil dryness that is not caused by, but rather associated with, high temperature due to reduced latent cooling [20]. This latter case represents a confounding influence of water supply stress that biases the estimated effect of a change in temperature [15]. This bias introduced into estimates of temperature stress on yields due to imperfect representation of water supply stress is shown analytically (Supplementary Discussion 1), simulated (Extended Data Fig. 1) and shown empirically (Fig. 2).

The temperature response is significantly different in the TS and TP models $(p=0.04$, pooled across all crops) and the TS model indicates a moderated effect of extreme heat on yields relative to the TP model (Fig. 2). The damage associated with exposure to a day with a maximum temperature of $40^{\circ} \mathrm{C}$, relative to one at $25^{\circ} \mathrm{C}$, is reduced in the TS model relative to the TP model for each crop (18\% for maize, $24 \%$ for soybeans, $72 \%$ for sorghum, and $24 \%$ for millet). The temperatures at which warming switches from benefiting to damaging yield is also generally higher in the TS than TP model $\left(22^{\circ} \mathrm{C}\right.$ to $24.5^{\circ} \mathrm{C}$ for maize, $24.6^{\circ} \mathrm{C}$ to $26.3^{\circ} \mathrm{C}$ 
for soybeans, and $27.1^{\circ} \mathrm{C}$ to $29.1^{\circ} \mathrm{C}$ for sorghum, but $19.6^{\circ} \mathrm{C}$ to $19.5^{\circ} \mathrm{C}$ for millet). The higher temperature associated with damage brings global empirical estimates into closer accordance with field, laboratory, and greenhouse experiments that find optimal temperatures for maize between $26.4^{\circ} \mathrm{C}$ and $30.8^{\circ} \mathrm{C}$, depending on growth stage [33]. Underestimation of the optimal temperature may persist even in the TS model due to measurement error of temperature or soil moisture, shortcomings in our empirical representation of these effects, or to differences between experimental and field conditions.

The estimated influence of temperature stress is moderated in the TS model relative to the TP model more at higher temperatures than at lower temperatures for two reasons (Supplementary Discussion 1 and Extended Data Fig. 1D-F.) First, yield is more sensitive to soil moisture in dry than wet conditions, and low soil moisture is correlated with high temperatures (Fig. 2, Fig. 1C, Extended Data Fig. 2A). Second, soil moisture and temperature are more strongly related in warm than cool conditions (Extended Data Fig. 2C).

Collectively, these findings show that a substantial portion of global crop damages that correlate with extreme heat are due to low water supply, consistent with previous analyses of U.S. crops $[9,19,14]$. These results also show that the influence of temperature stress on global agricultural yield remains substantial even after temperature-mediated influences on soil moisture have been accounted for, consistent with field studies showing depressed photosynthetic rate with high VPD even in well-irrigated conditions [34].

Comparing estimated effects in irrigated and non-irrigated fields supports the TS model. As an additional evaluation of the ability of the TS model to separate water supply and temperature stress, we estimate TS and TP models that allow the effects of temperature and soil moisture or precipitation to differ in irrigated and non-irrigated areas (Fig. 3, Methods). We find that the estimated damages of extreme heat are nearly identical in irrigated fields between TS and TP models $(\mathrm{p}=0.943$ for the null that the TP and TS model temperature curves are identical in the pooled model), but that the effect of extreme heat is exaggerated in non-irrigated areas in TP models relative to TS models $(\mathrm{p}=0.025)$. Analysis of crops individually gives consistent results (Extended Data Fig. 6). The finding of similar temperature effects in irrigated agriculture, where water supply stress is likely to be low, and different estimated temperature effects in unirrigated regions, where water supply stress is likely to be higher, indicates that TS models are indeed capturing and separating the effects of water supply from correlated temperature stress, whereas in TP models the influence of water supply is partially represented in the estimated temperature response. Furthermore, the influence of temperature is similar in irrigated and non-irrigated areas in the TS model ( $\mathrm{p}=0.17$ for the null that the influence of temperature is identical in irrigated and unirrigated areas in the pooled model) but not the TP model $(\mathrm{p}=0.03)$.

We note that it is theoretically possible that irrigation could attenuate damages of extreme heat by alleviating water supply stress if water supply has an interactive effect with temperature stress upon yield, as has been found for U.S. maize [9]. We find no evidence, however, that the level of soil moisture alters the influence of temperature, or vice versa, in a substantial or statistically significant way at the global scale for any of the four crops (Extended Data Fig. 7). This could be due either to differences in agricultural practices between the U.S. and the rest of the world, or to the limited sample size and thus power of 
the national-level sample to resolve potential interaction effects between water supply and temperature stress.

Collectively, these results suggest that some of the benefits of irrigation attributed to a reduction in the damages of extreme heat reported in the literature (e.g. [7, 32, 35]) instead result from attenuation of water supply stress. Understanding the mechanisms through which irrigation attenuates damages associated with high temperatures is critical for predicting how irrigation can limit damages from climate change.

Irrigation also modifies the influence of soil moisture on yield $(p<0.01$ for the null that the the response to soil moisture is identical in irrigated and non-irrigated areas in the pooled model). Consistent with a well-regulated water supply, irrigated areas show little benefit from increasing soil moisture at low to moderate levels, whereas unirrigated areas benefit from increased soil moisture at low to moderate levels, likely due to reduced water supply stress. At high levels of soil moisture, irrigated areas show substantial damages from increased soil moisture, whereas unirrigated areas show little effect. A similar pattern is found for crops individually when there is is a significant effect of irrigation on the influence of soil moisture (Extended Data Fig. 3). Yield-losses from high soil moisture in irrigated areas may stem from root-zone anoxia resulting in nutrient deficiencies and build up of toxic substances, from increased risk of disease, or from delays in planting or harvest [36, 37, 38, 39]. Another possibility involves naturally occurring high soil moisture limiting precise control of soil moisture through irrigation; for example, morning irrigation can facilitate daytime photosynthesis whereas evening precipitation can lead to leaf rot.

\section{Separation of water supply stress and temperature stress leads to more accurate} projections of the influence of climate change on crop yield due to differences between the historic correlation and future change in soil moisture and temperature. The average temperature during the growing season in cropped regions, as simulated by six earth system models from CMIP6, warms by $4.4^{\circ} \mathrm{C}$ between $2015-2035$ and 2080 2100 in the SSP5-8.5 experiment (Fig. 1). These same regions dry, on average, by -0.0064 $\mathrm{cm}^{3} \mathrm{~cm}^{-3}$. Applying our TS model to these simulations at the daily, grid-scale level indicates a decreases in global-average production of all crops (-20\% for maize, $-32 \%$ for soybean, $-9 \%$ for sorghum, and $-17 \%$ for millet, Fig. 4). The vast majority of projected climate damages to global average yield in the TS model stem from heat stress $(-19 \%$ for maize $-31 \%$ for soybeans $-9 \%$ for sorghum and $-18 \%$ for millet), whereas soil moisture plays a variable and smaller role $(-1 \%$ for maize, $-2 \%$ for soybeans, $0 \%$ for sorghum, and $1 \%$ for millet, Extended Data Fig. 9). Note that all projections in this analysis are not predictions of future yield because they consider only future changes in water availability and temperature stress, and not other determinants of yield such as agricultural technology or $\mathrm{CO}_{2}$.

We check the paired CMIP6 - TS model simulations by comparing the variability of predicted yield anomalies in the historic sample to those predicted using CMIP6 data from 2015-2035. We find that the paired CMIP6 - TS model replicates the finding in the observed sample from 2007-2018 of relatively equal contribution to yield variability due to temperature and soil moisture (50\% for soil moisture versus $40 \%$ for temperature and $10 \%$ for their covariance for maize, $19 \%$ versus $44 \%$, and $37 \%$ for soybeans, $55 \%$ versus $13 \%$, and $32 \%$ for sorghum, and $32 \%$ versus $30 \%$, and $38 \%$ for millet). Contribution to yield anomalies is 
reported as the variance of yield anomalies due to the temperature or soil moisture component of the TS model as well as their covariance, which sum to the variance of yield anomalies predicted by the full TS model. Further, projections of impacts on global agricultural yield from climate change using the empirical TS model are consistent with projections using an ensemble of process-based crop models, which show climate-induced damages to yield, omitting $\mathrm{CO}_{2}$ fertilization effects, of $-30.2 \%$ for maize and $-33 \%$ for soybeans from 2069-2099 compared to $-20 \%$ and $-32 \%$ in our projections [40]. Larger simulated effects using these process-based models for maize may in part be due to a larger simulated warming of $5^{\circ} \mathrm{C}$ over global croplands compared to $4.4^{\circ} \mathrm{C}$ in our sample.

Projections using TP models indicate damages that are larger than the TS model for each crop ( $28 \%$ higher for maize, $28 \%$ for soybeans, $320 \%$ for sorghum, and $48 \%$ for millet). The null hypothesis of equal damage for the TS and TP models is rejected ( $p<0.01$ for each of the four crops using a paired t-test). This higher estimate occurs because projected warming is associated with less drying than would be expected based on observed synoptic correlations. Historically, a $4.4^{\circ} \mathrm{C}$ hotter growing season is on average $-0.051 \mathrm{~cm}^{3} \mathrm{~cm}^{-3}$ drier, an order of magnitude more than the corresponding drying from climate change (Methods). Given the greater skill of the TS model than the TP model, our findings in irrigagted versus unirrigated areas, and the historic and projected correlations between growing season temperature and soil moisture, we conclude that the TP model overestimates future yield damages because of implicitly predicting water supply stress that is proportional to historical covaration with temperature.

Climatological divergence in the association between temperature and soil moisture is unsurprising given that the historic covariation is driven by synotpic variations, such as atmospheric blocking patterns that give rise to dry and hot high-pressure systems. Long-term climate change, in contrast, involves a warming atmosphere that comes into a new moisture equilibrium with the oceans and is associated with a global-average increase in precipitation. Growing season average daily precipitation increases by $0.36 \mathrm{~mm}$ across cropped regions in the SSP5-8.5 simulations that we consider, and is also simulated to increase in seasons prior to the growing season [41], thereby recharging soil moisture.

The finding of reduced projected global climate change damages when modeling water supply directly in TS models relative to TP models is consistent with one study of U.S. agriculture [9], but differs from another, where no difference was found across crops [8], potentially due to heterogeneous global agricultural practices and highlighting the importance of a global analysis. The finding that future damages are largely determined by temperature stress is consistent with previous studies of agriculture in the U.S. [8] and with international analyses of unmanaged vegetation [23].

Findings for global average crop yields hold regionally, with a few exceptions (Fig. 4). Changes in temperature and soil moisture from climate change reduce yields for all crops in each of the fourteen IPCC AR5 regions analyzed except for sorghum in Central Europe and East Asia (Extended Data Fig. 8). Temperature accounts for the majority of this damage other than for sorghum in six of the fourteen regions (Extended Data Fig. 9). Projected damages across regions from changes in soil moisture are relatively small and inconsistent in sign. There are, however, some important exceptions where reductions in soil moisture are projected to substantially decrease yields of some crops including in Central America and the Mediterranean (Fig. 4). Furthermore, although the projected global and regional mean in- 
fluences of changes in soil moisture are small, such averaging masks substantial heterogeneity and across-model uncertatinty in locations such as Central America, Western Africa, and India, as demonstrated by pixel-wise minimum and maximum effects across models (Extended Data Fig 10). These regional patterns in the influence of soil moisture and temperature reflect the relatively consistent increases in temperature across space and less consistent drying across space (Fig. 1), with contributions also stemming from the nonlinearity of the temperature and soil moisture response functions (Fig. 2). Comparing regional projected damages using the TS and TP models, we find that the TP model overestimates damages in every region for all four crops, with agreement across climate models (Extended Data Fig. $8)$.

\section{Discussion}

The importance of measuring and modelling soil moisture for accurately predicting the effects of climate change on global yield indicates that remaining deficiencies in our observations and simulations of soil moisture are concerning. Our satellite-based estimates of root-zone soil moisture depend on a simple percolation model forced by surficial observations (Methods). Improved spatial and temporal resolution of measurements as well as measurements of soil moisture at different and deeper depths would further improve estimations of short and long-run influences of water supply on crop yields. The fact that we still find maize damages from increasing temperatures at values below the optimum found in field studies with well-watered cultivars (e.g. [42]) suggests that we may have only partially accounted for variations in water supply.

Comparisons of present-day simulated and observed soil moisture show large biases in simulated growing season climatologies (Extended Data Fig. 11) and large variability across models (Extended Data Fig. 12). Imperfect simulation of current soil moisture distributions calls into question the accuracy of projected changes in soil moisture, especially regarding at what values of soil moisture warming or drying may occur. To test the sensitivity of our results to such potential errors we debiased the CMIP6 daily maximum temperature, soil moisture, and precipitation data to match the location-specific mean (Extended Data Figs 13) of observations [43] and find remarkably stable projected effects. Results are also similar when debiasing both the mean and variance of simulations (Extended Data Figs 14). Our debiasing, however, only partially corrects errors in the CMIP6 simulated values given nonlinear and dynamic relationships between temperature, soil moisture, and precipitation. Improvements in climate model representations of soil moisture are needed to better constrain the effects of climate change on global agriculture.

Inclusion of other environmental determinants of yield into empirical crop models such as sunlight, wind speed, leaf temperature may also enable a more nuanced understanding of water supply stress, temperature stress, and their interactions with other environmental factors $[21,44]$. Consideration of $\mathrm{CO}_{2}$ concentration could also alter the influences of water supply and temperature stress as well as projected yield, especially for crops using the C3 photosynthetic pathway such as soybeans [40]. Recent efforts to incorporate $\mathrm{CO}_{2}$ effects into empirical crop models are promising [45], and present an important avenue for future work. Further, finer-resolution measurements of crop yield would enable more complex models of how water supply and temperature stress influence yield that are intractable with nationallevel data, including resolving within-growing-season heterogeneity [8] or interaction effects 
between water supply and demand [9].

Collectively, our results demonstrate that an empirical model jointly accounting for temperature and soil moisture better captures and separates the influences of water supply and temperature stress on global crop yields. This separation improves the accuracy of historical and future predictions of climatic influences on yield. Our results indicate a need for substantial emissions mitigation and agricultural adaptation to lessen climate-induced damages to agricultural productivity, especially related to temperature stress. Our analysis also suggests that re-examining the influence of water supply, temperature stress and climate change on other outcomes with potential agricultural mechanisms, such as conflict, migration, or health may provide novel insights. 


\section{Methods}

Data The European Space Agency (ESA) Climate Change Initiative Plus Soil Moisture Project v06.1 dataset compiles active and passive satellite measures of surface soil moisture up to a depth of approximately $5 \mathrm{~cm}$ at daily $0.25^{\circ}$ by $0.25^{\circ}$ resolution $[26,27]$. We estimate root zone soil moisture using an auto-regressive exponential framework, following [9]. Similar results are obtained using the raw values themselves likely due to the relatively strong correlations between surface and root zone $(\approx 1 \mathrm{~m})$ soil moisture [46].

The Climate Prediction Center compiles and interpolates station measures to produce a gridded daily maximum surface air temperature and daily precipitation product with $0.5^{\circ}$ by $0.5^{\circ}$ resolution from 1979 -present $[47,28]$. We use daily maximum rather than mean temperature to better approximate the vapor pressure deficit and to capture extreme heat stress.

The Food and Agricultural Organization of the United Nations provides annual measures of maize, soybean, millet and sorghum yield at the national level [29]. To match the national yield data to the distribution of growing season climate data we take the cropped-fractionweighted mean of each variable's linear and nonlinear feature values over year-2000 cropped area [48] and the growing season [49] using a methodology similar to previous studies [1, 30]. When aggregating to the growing season, we calculate all non-linear feature expansions (e.g. $\left.x, x^{2}\right)$ before averaging spatially and temporally to appropriately recover nonlinearities in the response [1]. We average the soil moisture data to $0.5^{\circ}$ by $0.5^{\circ}$ resolution to match the resolution of the temperature and precipitation data before calculating nonlinearities to facilitate comparison between measures of water supply.

We limit our sample to the years 2007-2018 since the frequency of soil moisture measurements increased substantially in 2007 due to additional satellites making observations [50]. Findings are consistent though less precise using the full time series from 1980 to 2018 due likely to infrequent measurements of soil moisture providing a less precise measure of the growing season distribution. Similarly, we include only observations where daily temperature, precipitation and soil moisture were observed at least every other day within the growing season to reduce measurement error of growing season conditions. To reduce noise in the outcome variable, we drop observations with demeaned and detrended yield anomalies greater than 4 standard deviations away from the mean; results are consistent when including these observations, which account for less than 1 percent of the data.

Yield Model We model the influence of daily temperature and soil moisture on yields of a given crop using a panel fixed effect regression model [30]:

$$
Y_{i t}=f_{T}\left(T_{i t}\right)+f_{S}\left(S_{i t}\right)+\lambda_{i}+\alpha_{i} t+\epsilon_{i t}
$$

where $Y_{i t}$ are $\log$ yields in a country $i$ during year $t ; f_{T}($.$) and f_{S}($.$) are restricted cu-$ bic spline expansions of daily maximum temperature and soil moisture during the growing season; quadratic polynomial expansions give consistent qualitative results though generally lower predictive performance (Extended Data Table 1). TP models substitute $f_{S}($.$) with$ a restricted cubic spline expansion of daily precipitation; TSP models include $f_{T}(),. f_{S}($. 
and $f_{P}($.$) . We also evaluate the performance of models that estimate yields as a function of$ nonlinear growing season average conditions rather than daily conditions, and generally find lower performance, especially for temperature (Extended Data Table 1). Seasonal precipitation tends to show higher performance than daily precipitation though still lower than soil moisture.

The country-specific fixed effects, $\lambda_{i}$ account for any time-invariant differences between countries such as soil type, and the country-specific time trends, $\alpha_{i} t$ account for countryspecific trending variables such as technological adoption. The residual, $\epsilon_{i t}$, captures all remaining factors that vary over time within countries but are uncorrelated with the modeled climate variables. We compute standard errors clustering by country, which allows for arbitrary patterns of serial correlation within a country over time [30].

Model fit is evaluated using 10-fold cross validation within $\mathrm{R}^{2}$, which measures the outof-sample predictive performance after the country-specific intercepts and trends have been removed from the data. We split the data randomly into training and validation folds for evaluation; cross-country training and validation splits show consistent results (Supplementary Discussion 2, Supplementary Table 2). We evaluate full model fit, including intercepts and trends, using the adjusted $\mathrm{R}^{2}$, which takes account of the number of parameters used to estimate the model.

Given the similarity in response to environmental factors across crops, we estimate a pooled model, which estimates a common response to temperature and soil moisture across crops, $c$ :

$$
Y_{i t c}=f_{T}\left(T_{i t c}\right)+f_{S}\left(S_{i t c}\right)+\lambda_{i c}+\alpha_{i c} t+\epsilon_{i t c}
$$

Country-crop time series are still effectively demeaned and detrended individually, as in Eqn. 1 , by the country-crop fixed effects and time trends, $\lambda_{i c}+\alpha_{i c} t$. Standard errors are calculated allowing for arbitrary serial correlation within each country-crop time series.

To allow irrigation to modify the influence of environmental factors (Fig. 3), we interact $f_{T}($.$) and f_{S}($.$) with the fraction of crop-specific cropped area in each country equipped for$ irrigation. We obtain area equipped for irrigation at 5 minute spatial resolution for the year 2005 from the Global Map of Irrigation Areas version 5 [51]. We calculate the fraction of cropped area equipped for irrigation for each crop at the $0.5^{\circ}$ by $0.5^{\circ}$ resolution, and winsorize the value to 1 . The value exceeds 1 in a small number of grid cells because the area equipped for irrigation data is not crop-specific, so the area equipped for irrigation can exceed the cropped area. We interact the cropped-fraction-equipped-for-irrigation values with the temperature, soil moisture and precipitation values, along with their nonlinear feature expansions before spatially aggregating to the national level. While the area equipped for irrigation data is not crop-specific, aggregating values to the national level weighting by crop-specific cropped areas differentiates area equipped for irrigation by crop at the national level.

In our primary irrigation model (Fig. 3) we pool the influence of temperature and soil moisture across crops due to both similarity of responses to environmental factors and because the irrigation data does not specify what type of crop is irrigated.

$$
Y_{i t c}=f_{T}\left(T_{i t c}\right)+f_{S}\left(S_{i t c}\right)+f_{T}\left(T_{i t c} I_{i c}\right)+f_{S}\left(S_{i t c} I_{i c}\right)+\lambda_{i c}+\alpha_{i c} t+\epsilon_{i t c}
$$


We note that any differences in average yield associated with differences in irrigated fraction across countries will be accounted for in the country-crop fixed effect, $\lambda_{i c}$. This model estimates heterogeneous effects of environmental factors on yield by comparing effects over time in countries with more or less cropped area equipped for irrigation.

We estimate crop-specific irrigation models using the same functional form, but model the response individually for each crop (Extended Data Fig 6):

$$
Y_{i t}=f_{T}\left(T_{i t}\right)+f_{S}\left(S_{i t}\right)+f_{T}\left(T_{i t} I_{i}\right)+f_{S}\left(S_{i t} I_{i}\right)+\lambda_{i}+\alpha_{i} t+\epsilon_{i t}
$$

Models estimating heterogeneous impacts of temperature and precipitation have the same form, but with functions of precipitation replacing functions of soil moisture.

To test whether the level of soil moisture modifies the influence of temperature, and vice versa, we estimate a model interacting the nonlinear responses of temperature and soil moisture (Extended Data Fig. 7):

$$
Y_{i t}=f_{T}\left(T_{i t}\right)+f_{S}\left(S_{i t}\right)+f_{T S}\left(T_{i t} S_{i t}\right)+\lambda_{i}+\alpha_{i} t+\epsilon_{i t}
$$

As in the primary specification, Eqn 1, we model $f_{T}($.$) and f_{S}($.$) with restricted cubic$ splines. We model the interaction between temperature and soil moisture, $f_{T S}($.$) , with a$ quadratic polynomial (i.e. $T S+T^{2} S+T S^{2}+T^{2} S^{2}$ ). As with other nonlinearities, interactions are calculated at the daily $0.5^{\circ}$ by $0.5^{\circ}$ resolution before aggregating to the growing season and country.

We calculate the amount of drying for a given amount of warming in the observed historical data by regressing national detrended anomalies in growing season average soil moisture against national detrended anomalies in growing season average temperature, pooled across crops.

Projections To project future changes in yield due to climate change, we apply empirical crop models to simulations of future climate from CMIP6 [31] from 2015-2035 to 20802100 under the SSP5-8.5 scenario for the models GFDL-CM4, CanESM5, IPSL-CM6A-LR, MIROC6, MRI-ESM2-0, and BCC-CSM2-MR. We selected all models from the google cloud CMIP6 archive with available daily surface soil moisture (mrsos), maximum temperature (tmax), and precipitation (pr) in the historical (2000-2015), present (2015-2035) and future (2080-2100) periods. We regrid outputs of each model to a common $0.5^{\circ}$ by $0.5^{\circ}$ resolution using bi-linear interpolation to match the resolution of the temperature, soil moisture and precipitation observations.

We calculate nonlinear expansions of the daily soil moisture, maximum temperature and precipitation values from CMIP6 for each crop in the same way as for observations. We pass these representations of the future distribution of growing season temperature, soil moisture, and precipitation through the TS and TP empirical yield models for each crop to get projections of future yields. Subtracting projected present day yields (2015-2035 average) from projected future yields (2080-2100 average) gives the change in yields due to climate change induced changes in temperature, soil moisture and precipitation. When calculating averages across regions or globally, we weight by cropped-fraction. 
To test the sensitivity of our results to biases due to imperfect simulation of temperature, soil moisture and precipitation in the CMIP6 models, we debias the CMIP6 data relative to observations and replicate our projections (Extended Data Fig. 11). We employ two different debiasing methods. First, we employ a mean-debiasing approach by calculating the mean difference between CMIP6 simulated (2000-2015) and observed (2007-2018) values in each grid cell during the historical period, and correcting the CMIP6 values accordingly for each CMIP6 model. For temperature, we add the difference between the historical observed and historical simulated data to the CMIP6 values in each grid cell. For soil moisture and precipitation, which have a minimum value of zero, we scale the simulated values by the ratio of historical observed and historical simulated means [43]. Second, we employ a pixel-wise mean and variance debiasing approach. We debias the variance of the CMIP6 simulations by demeaning the values at each pixel, scaling the values by the ratio of the standard deviation of observed values to the standard deviation of simulated values, and adding the mean back in. We then mean-debias the CMIP6 data as in the first approach. We winsorize debiased values for soil moisture to a maximum of 0.5 and values for precipitation to a minimum of zero, to correct a small number of negative values that were introduced during the variance debiasing step. 


\section{Figures}
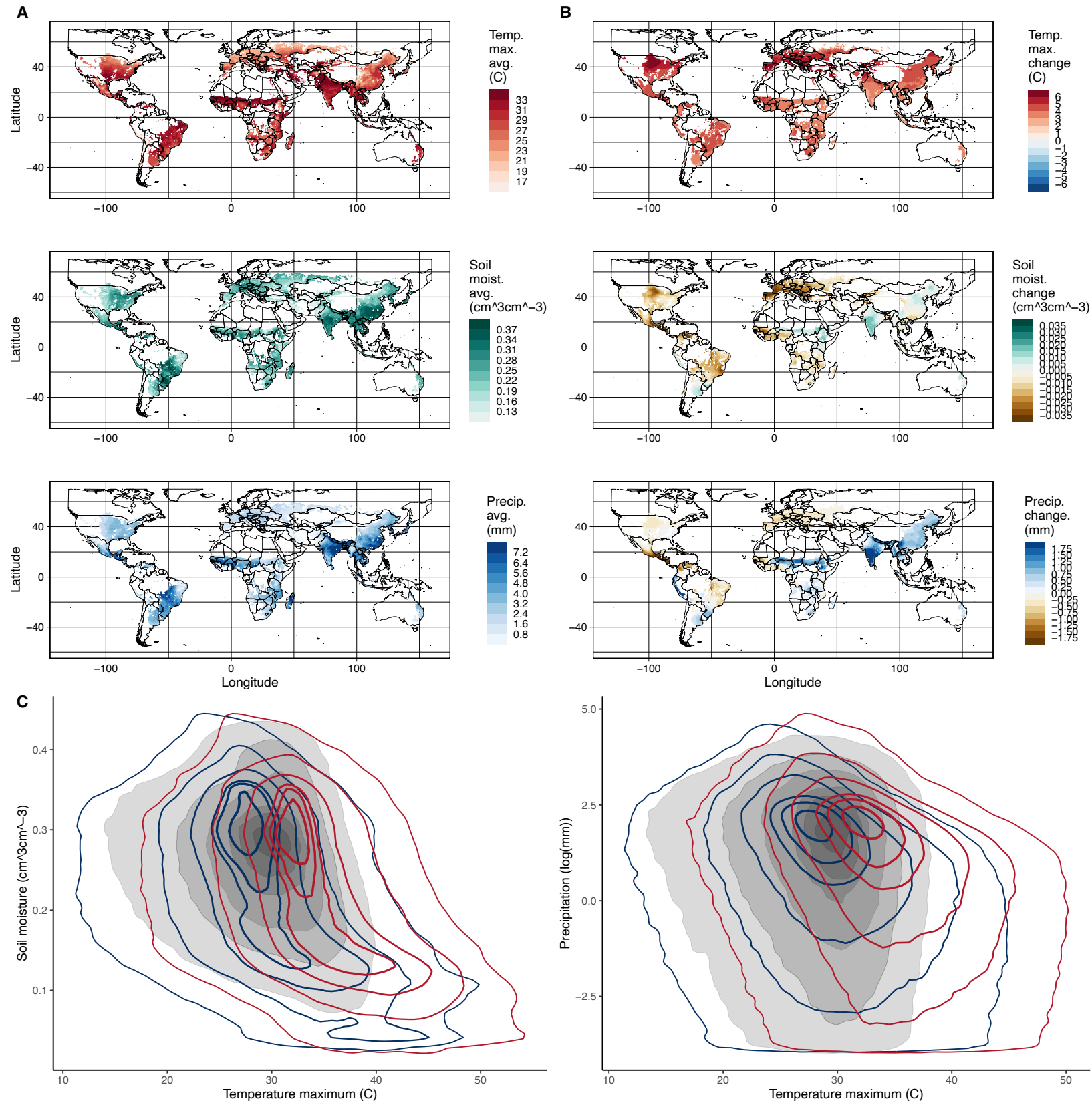

— Observations - CMIP6 Historical (2000-2015) - CMIP6 SSP585 (2080-2100)

Figure 1: Climate change alters the coupling of temperature, soil moisture and precipitation. (A) Observations of growing season cropped-fraction weighted average daily maximum temperature, root zone soil moisture and precipitation from 2007-2018 for maize, soy, millet and sorghum. (B) Projected change in temperature, soil moisture and precipitation from climate change (2015-2035 to 2080-2100 following SSP5-8.5, Methods). (C) The joint cropped-fraction-weighted growing season distribution of observed past (2007- 2018, grey), simulated past (2000-2015, black) and simulated future (2080-2100, red) daily temperature and soil moisture and temperature and precipitation. Simulated values are the average over six CMIP6 models (Methods). 

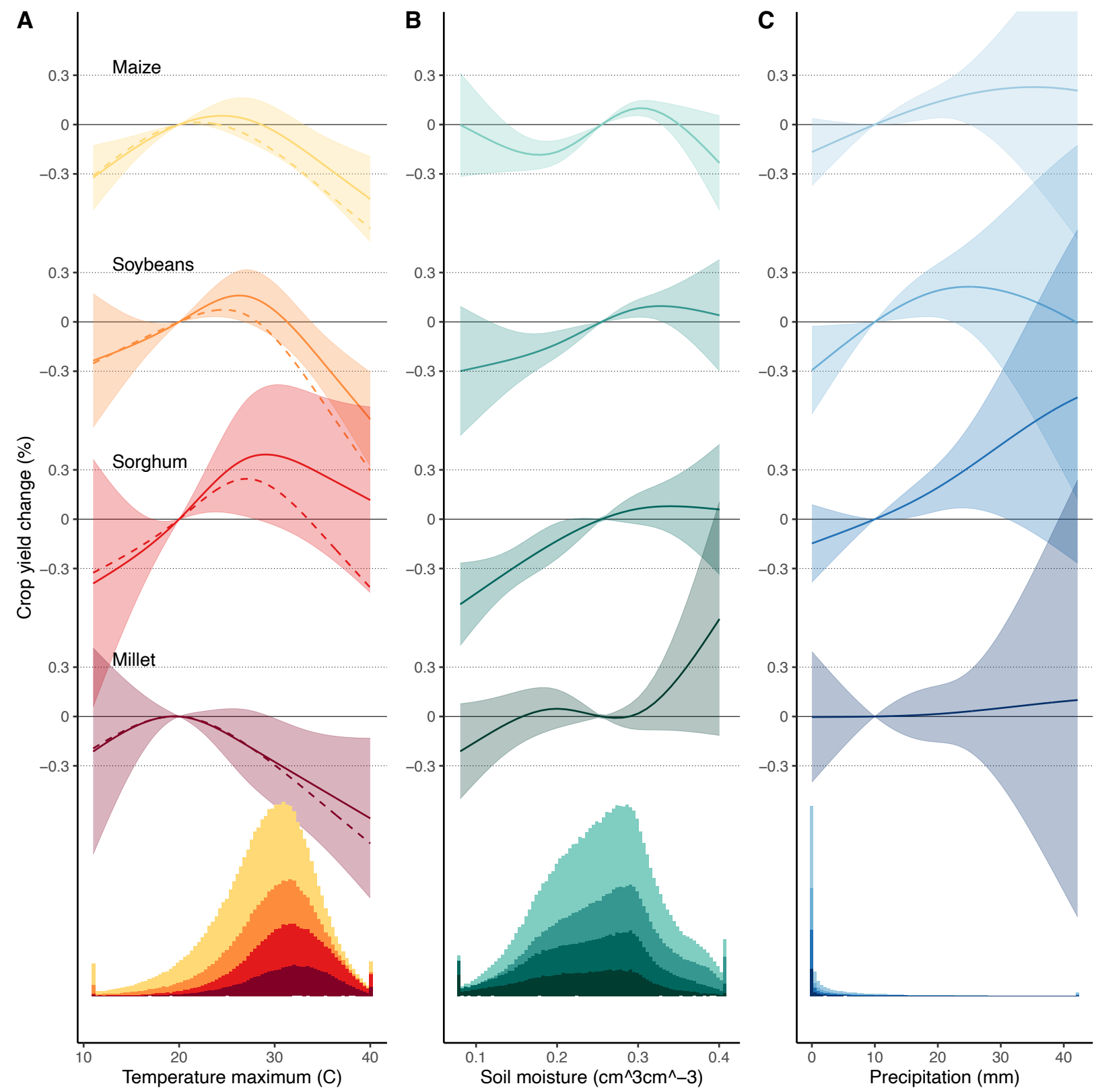

Figure 2: Modelling soil moisture and temperature jointly partitions the influence of water supply from that of temperature stress on global crop yields. (A) The response of yield to changing a day in the growing season from $20^{\circ} \mathrm{C}$ to a given temperature accounting for soil moisture (solid) is less negative than the same response estimated accounting for precipitation (dashed) across all four crops. (B) The response of yield to changing soil moisture for a day in the growing season from $0.25 \mathrm{~cm}^{3} \mathrm{~cm}^{-3}$ to a given value. (C) The response of yield to changing precipitation for a day from $10 \mathrm{~mm}$ to a given value. Shading shows the $95 \%$ confidence interval for each response, estimated accounting for serial correlation within countries over years. Histograms show the daily distribution of maximum surface air temperature, root zone soil moisture and precipitation over cropped areas, with outliers winsorized for display to their 0.5 and 99.5 percentiles for temperature and soil moisture and 1 and 99 percentiles for precipitation. 

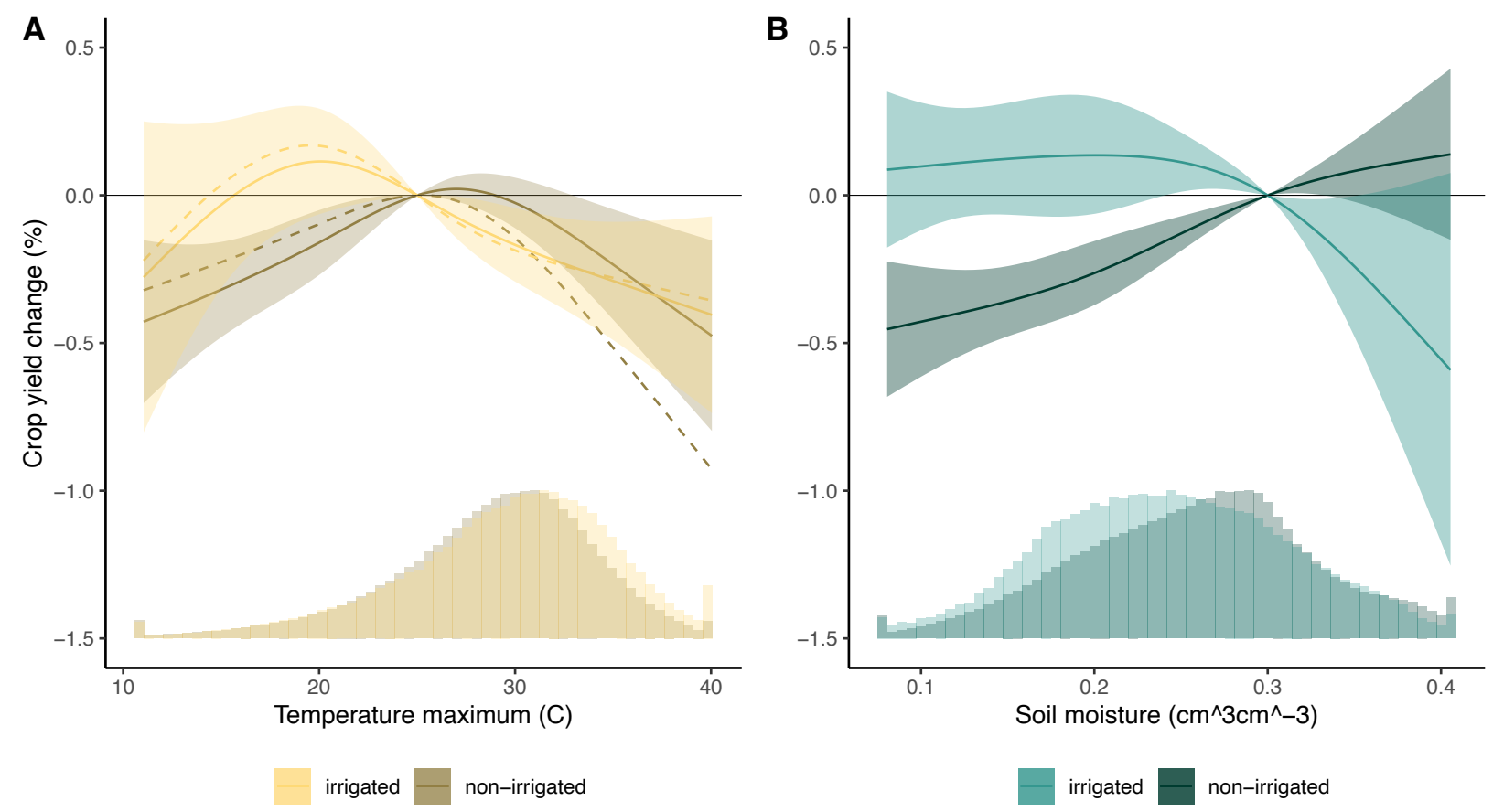

Figure 3: Irrigation modifies the influences of climatic factors on crop yield and validates empirical separation of water supply and temperature stresses. (A) The influence of daily maximum surface temperature on crop yield in irrigated (light) and non-irrigated (dark) areas for the TS (solid) and TP (dashed) models (Methods). (B) The influence of daily root zone soil moisture on crop yield in irrigated (light) and non-irrigated (dark) areas for the TS model. Values indicate the response of yield to changing a day in the growing season from $25^{\circ} \mathrm{C}$ or $0.3 \mathrm{~cm}^{3} \mathrm{~cm}^{-3}$ to a given value. Influences of climatic factors are pooled across maize, soybeans, sorghum and millet for statistical power; individual estimates are shown in Extended Data Fig 6. Shading shows the 95\% confidence interval for the estimated response of the TS model. Histograms show the cropped-fraction weighted growing season distribution of daily temperature and soil moisture in irrigated (light, greater than $50 \%$ of cropped area in pixel equipped for irrigation) and non-irrigated (dark, less than $50 \%$ of cropped area in pixel equipped for irrigation) areas. Allowing for irrigation increases the cross-validated within- $\mathrm{R}^{2}$ from 0.061 to 0.066 . 

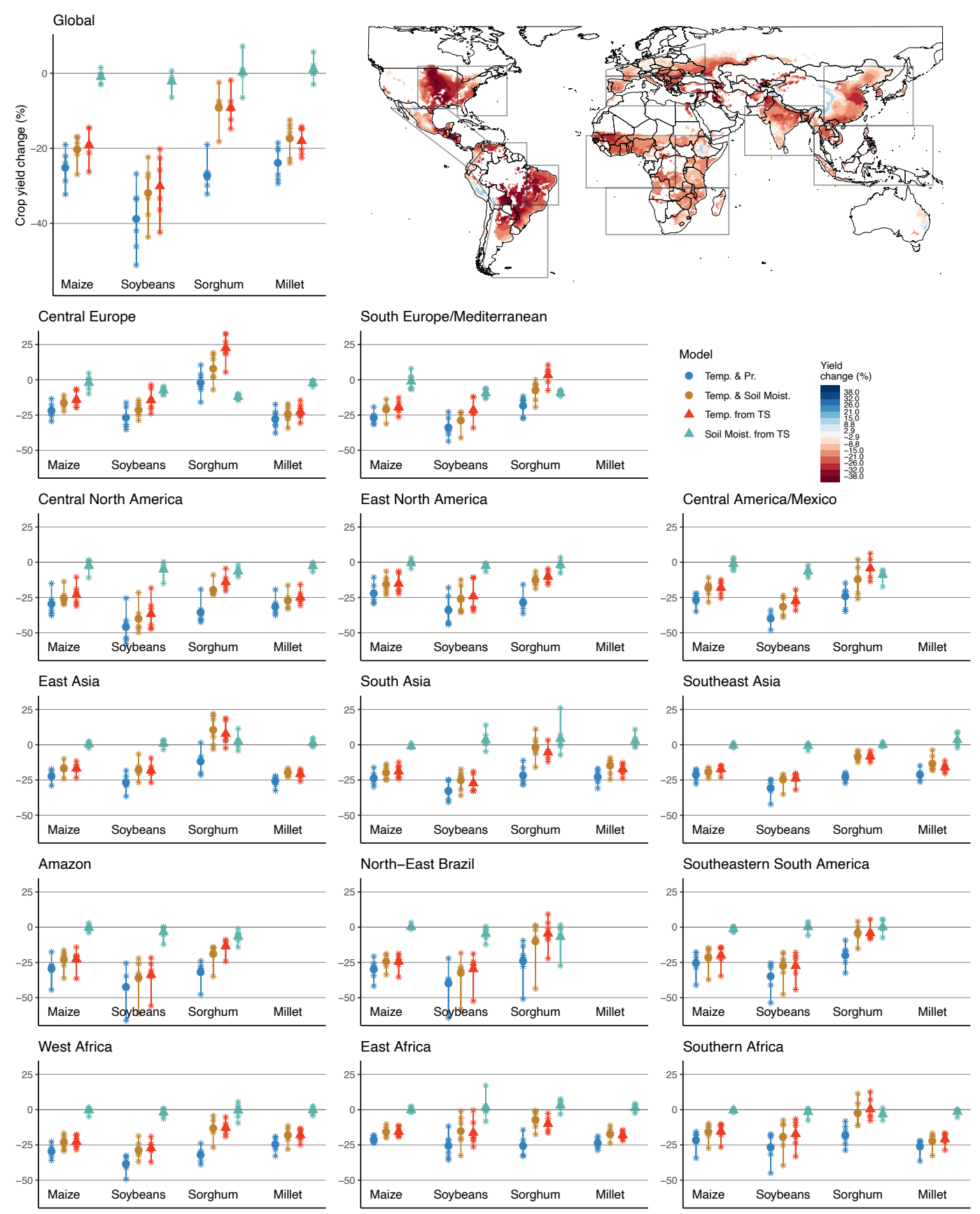

Figure 4: Projected influence of climate change on crop yield accounting for changes in water supply and temperature stress. Charts show the projected influence of climate change on cropped-fraction-weighted average yield using TP (blue) and TS (orange) empirical models. The total effect of the TS model is decomposed into an effect due to changes in temperature stress (red) and water supply stress (turquoise). Effects are averaged globally (top) as well as across the 14 IPCC AR5 reference regions with the highest cropped area. Stars show the average for each CMIP6 model and circles show the mean across models. To aid visual interpretation, lines show the range of means across models. The map shows the cropped-fraction weighted average influence of climate change using the TS model. IPCC AR5 reference regions are overlaid. Projections compare 2015-2035 to 2080-2100 using six CMIP6 models following SSP5-8.5. 


\section{Extended Data}

\begin{tabular}{|c|c|c|c|c|c|c|c|c|c|}
\hline & Crop & Model & Resolution & $\mathrm{T}$ & $\mathrm{P}$ & $\mathrm{S}$ & $\mathrm{TP}$ & $\mathrm{TS}$ & TSP \\
\hline 1 & Maize & quadratic & day & 0.057 & 0.021 & 0.055 & 0.062 & 0.073 & 0.072 \\
\hline 2 & Maize & quadratic & season & 0.022 & 0.028 & 0.056 & 0.066 & 0.076 & 0.078 \\
\hline 3 & Maize & cubic spline & day & 0.062 & 0.021 & 0.068 & 0.065 & 0.095 & 0.094 \\
\hline 4 & Maize & cubic spline & season & 0.020 & 0.026 & 0.067 & 0.068 & 0.092 & 0.090 \\
\hline 5 & Maize & irrigation & day & 0.097 & & 0.106 & 0.069 & 0.108 & \\
\hline 6 & Maize & interaction & day & & & & & 0.090 & \\
\hline 7 & Soybeans & quadratic & day & 0.096 & 0.034 & 0.093 & 0.093 & 0.122 & 0.112 \\
\hline 8 & Soybeans & quadratic & season & 0.048 & 0.059 & 0.092 & 0.109 & 0.123 & 0.119 \\
\hline 9 & Soybeans & cubic spline & day & 0.104 & 0.037 & 0.089 & 0.099 & 0.129 & 0.116 \\
\hline 10 & Soybeans & cubic spline & season & 0.043 & 0.056 & 0.088 & 0.118 & 0.128 & 0.126 \\
\hline 11 & Soybeans & irrigation & day & 0.141 & & 0.136 & 0.125 & 0.145 & \\
\hline 12 & Soybeans & interaction & day & & & & & 0.118 & \\
\hline 13 & Sorghum & quadratic & day & 0.015 & 0.009 & 0.042 & 0.018 & 0.045 & 0.043 \\
\hline 14 & Sorghum & quadratic & season & -0.004 & 0.015 & 0.041 & 0.024 & 0.045 & 0.042 \\
\hline 15 & Sorghum & cubic spline & day & 0.018 & 0.007 & 0.038 & 0.020 & 0.044 & 0.040 \\
\hline 16 & Sorghum & cubic spline & season & -0.008 & 0.015 & 0.038 & 0.027 & 0.045 & 0.038 \\
\hline 17 & Sorghum & irrigation & day & 0.034 & & 0.054 & 0.018 & 0.052 & \\
\hline 18 & Sorghum & interaction & day & & & & & 0.012 & \\
\hline 19 & Millet & quadratic & day & 0.029 & 0.005 & 0.019 & 0.027 & 0.026 & 0.025 \\
\hline 20 & Millet & quadratic & season & 0.024 & 0.005 & 0.021 & 0.026 & 0.028 & 0.026 \\
\hline 21 & Millet & cubic spline & day & 0.029 & 0.003 & 0.022 & 0.024 & 0.031 & 0.025 \\
\hline 22 & Millet & cubic spline & season & 0.028 & 0.002 & 0.016 & 0.024 & 0.025 & 0.022 \\
\hline 23 & Millet & irrigation & day & 0.028 & & 0.022 & 0.024 & 0.020 & \\
\hline 24 & Millet & interaction & day & & & & & 0.011 & \\
\hline
\end{tabular}

Extended Data Table 1: Model fit for a range specifications. Within $\mathrm{R}^{2}$ values are calculated using a 10-fold cross-validation procedure (Methods). Yield and explanatory climate factors are demeaned and detrended by country according to the model fixed effects prior to cross-validation. Column names indicate what combination of temperature (T), precipitation $(\mathrm{P})$ and soil moisture $(\mathrm{S})$ are included in the model. The model and resolution columns describe how nonlinear model features are calculated and at what temporal resolution the nonlinearities are applied. Restricted cubic splines calculated at the daily resolution are the primary specification. The irrigation model allows for heterogeneous effects in irrigated and non-irrigated cropland (Extended Data Fig. 6) and the interaction model allows the level of temperature to alter the influence of soil moisture and vice versa (Extended Data Fig. 7). 

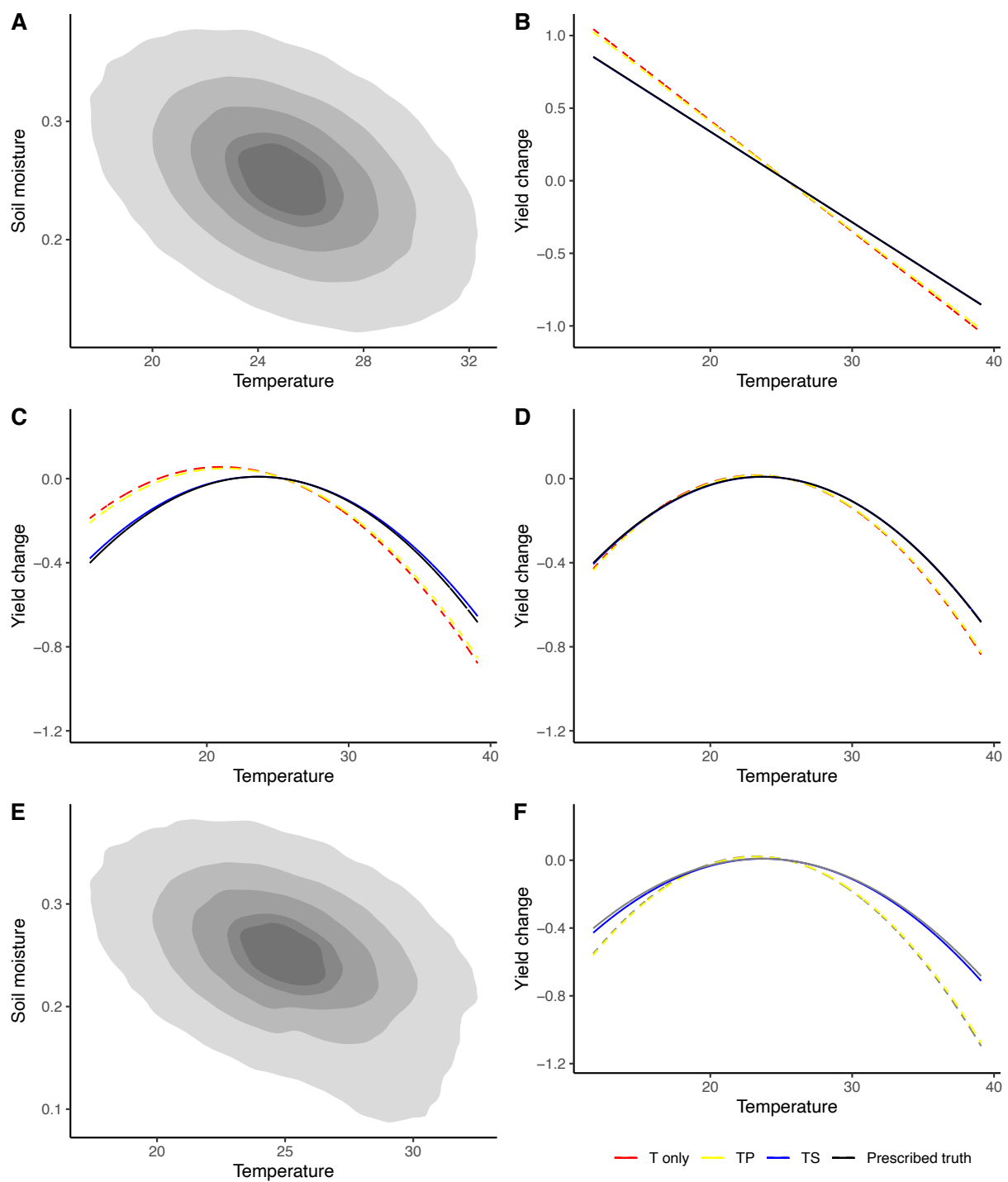

Extended Data Figure 1: Omitting measures of water supply biases estimated effects of temperature stress on crop yield in simulation. (A) Simulated daily temperature and soil moisture. (B) Estimated temperature response curves where simulated yields are a linear function of daily growing season temperature and soil moisture. The prescribed true response (black) is shown along with estimated responses modeling temperature (red), temperature and precipitation (yellow), and temperature and soil moisture (blue). (C) Similar response curves to $(\mathbf{A})$ except that yield is simulated and modeled as a quadratic, rather than linear, function of temperature. (D) Similar to (C) except that instead of soil moisture having a linear influence on yield, soil moisture has a nonlinear influence whereby increasing soil moisture at low values increases yield but increasing soil moisture at high values has little effect. This produces larger bias at high temperatures than at low temperatures in the models that omit soil moisture, which is consistent with what we observe in Fig. 2. (E) Similar to (A) but with a stronger simulated relationship between daily soil moisture and temperature in hot and dry conditions. (F) Similar to (D) but with additional bias at high temperatures relative to low temperatures due to the strengthened soil-moisture temperature relationship in $(\mathbf{E})$. 

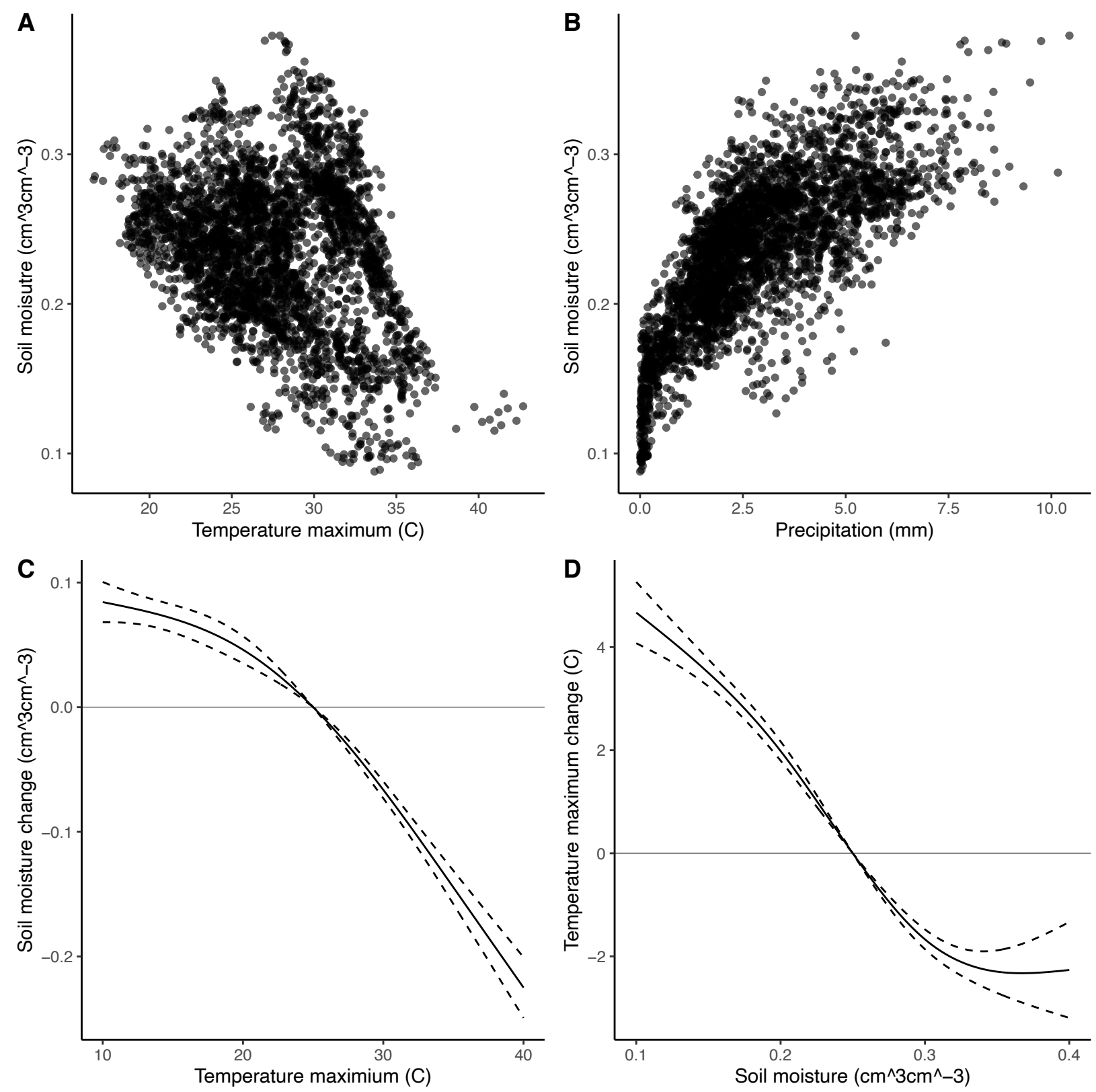

Extended Data Figure 2: Temperature and soil moisture are nonlinearly coupled during the growing season. (A) Growing season average maximum temperature plotted against growing season average soil moisture; each point represents an observation for a given country, crop, and year. (B) The same as A, but for growing season average precipitation and soil moisture $(\mathbf{C})$ The nonlinear relationship between daily maximum temperature and changes in soil moisture, accounting for country-specific intercepts and trends (left, within$\mathrm{R}^{2}=0.40$, effect is pooled across the four crops). (D) The nonlinear relationship between daily soil moisture and changes in maximum temperature, accounting for country-specific intercepts and trends (right, within- $\mathrm{R}^{2}=0.38$ ). The within- $\mathrm{R}^{2}$ explaining soil moisture as a nonlinear function of daily precipitation accounting for country-specific intercepts and trends is 0.40 . 

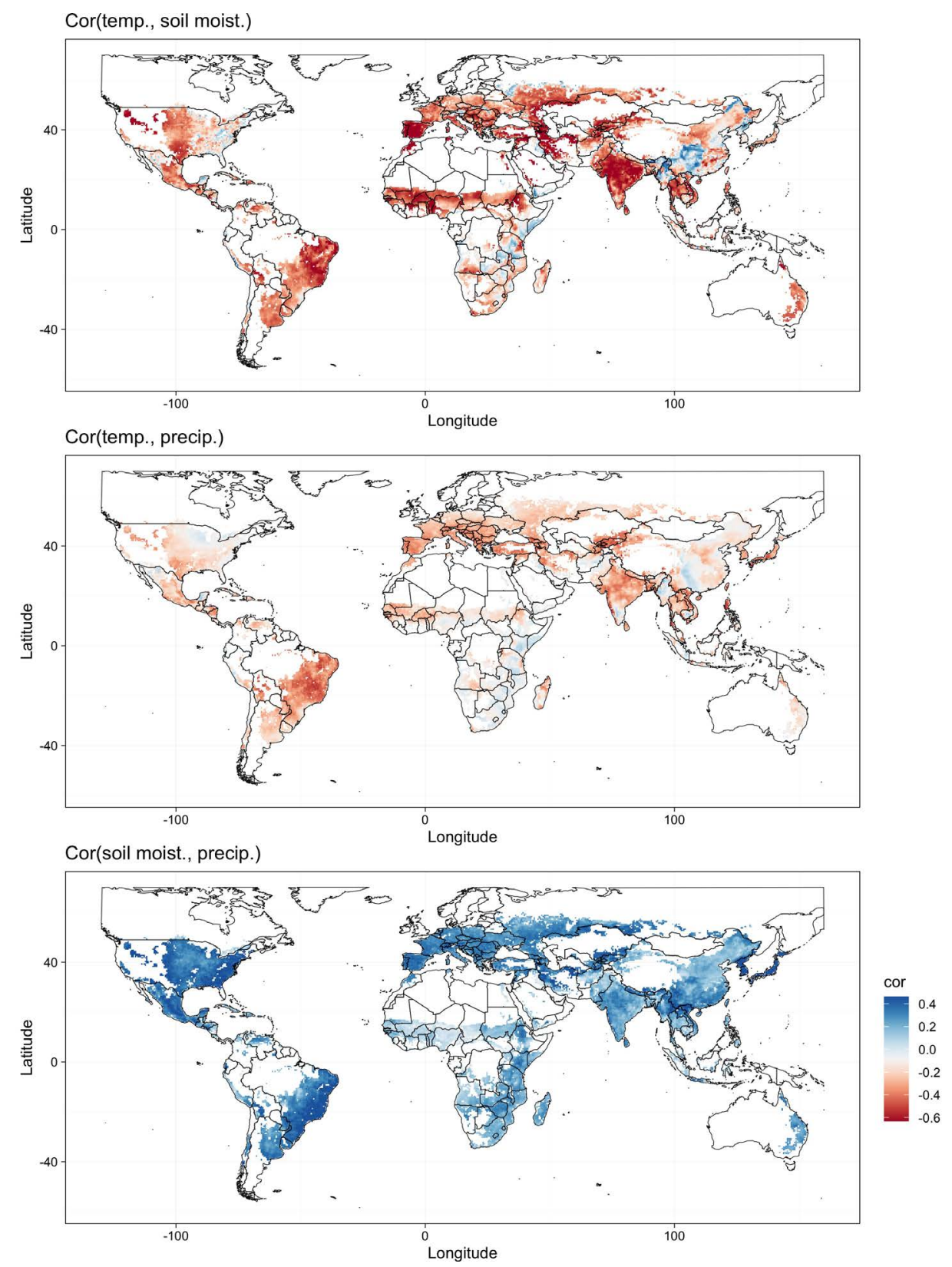

Extended Data Figure 3: Correlation of daily temperature, soil moisture and precipitation during the maize, soybean, sorghum and millet growing season over cropped areas. The Pearson's correlation coefficient is calculated weighting daily observations across crops by cropped fraction for each $25 \mathrm{~km}$ x $25 \mathrm{~km}$ pixel with harvest area greater than 100 hectares. 


\section{Maize}

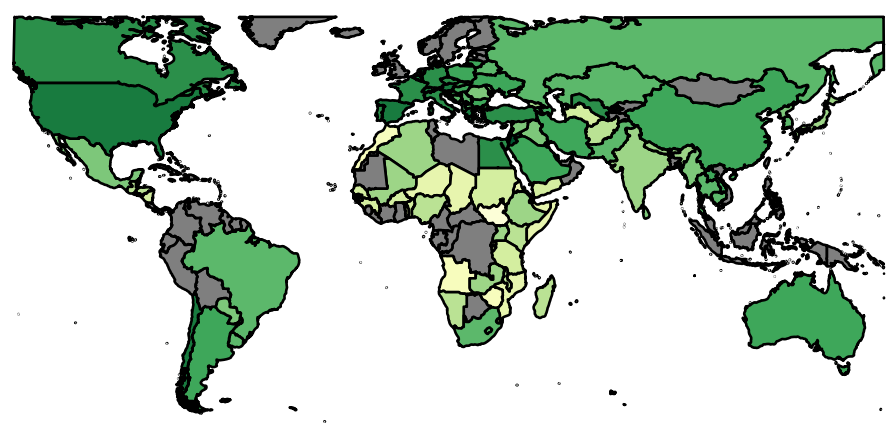

\section{Soybeans}

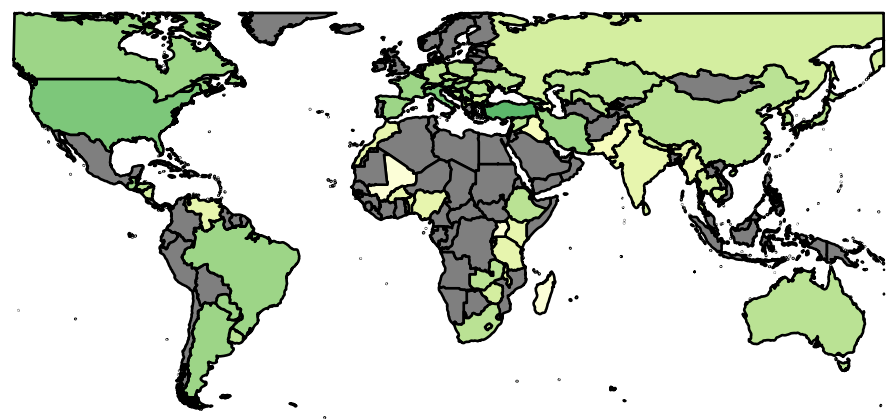

Millet

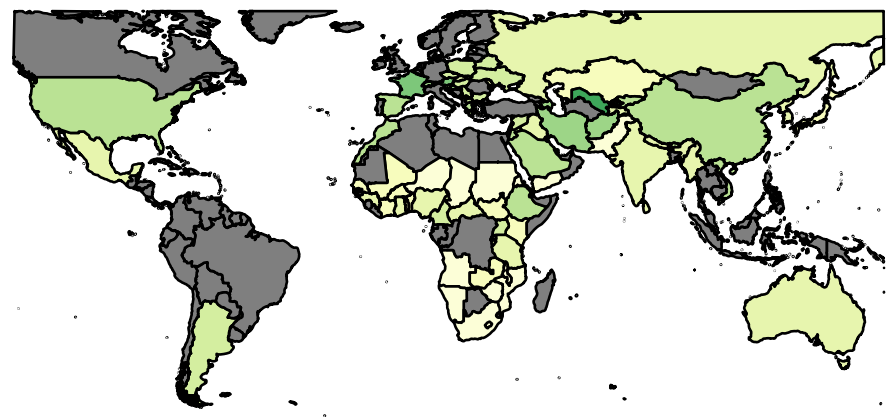

\section{Sorghum}

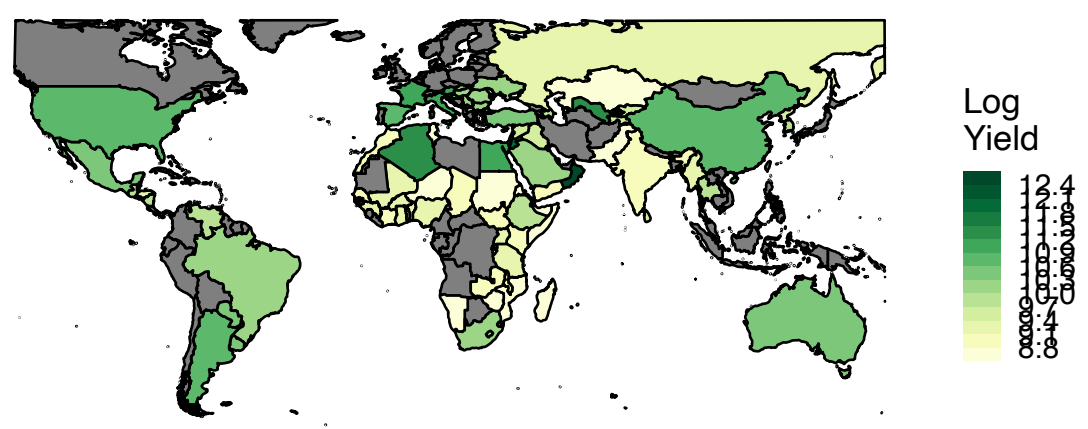

Extended Data Figure 4: Average yields of maize, soybeans, millet and sorghum. Average of yield data (2007-2018, hg/ha) from the Food and Agricultural Organization of the United Nations [29]. 

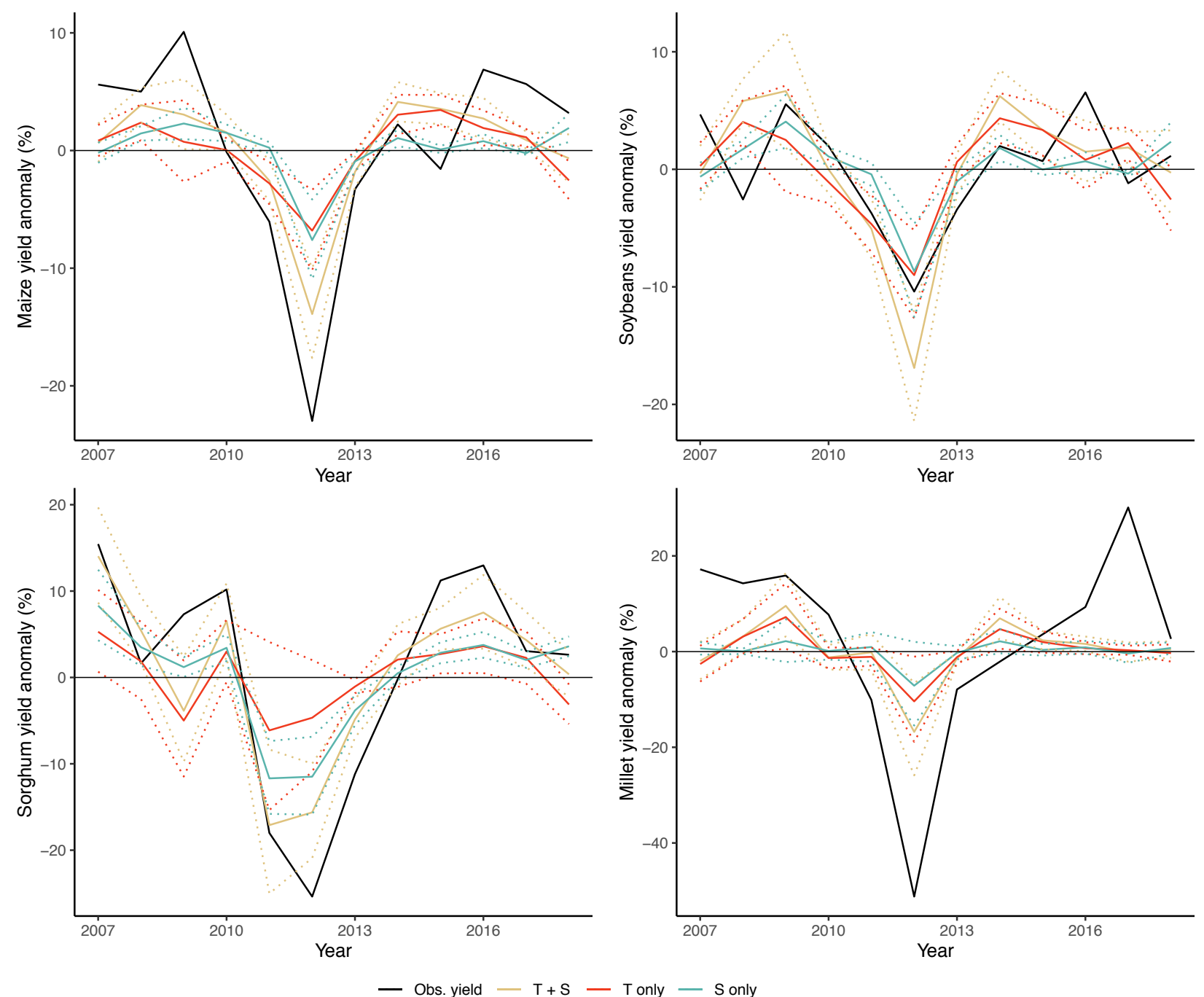

Extended Data Figure 5: Reconstructions of U.S. yield anomalies using a model accounting for water supply and temperature stress. Observed yield anomalies from the trend, along with predicted climate-induced yield anomalies from the trend using the TS model. The individual temperature and soil moisture components of this prediction are also plotted. $95 \%$ confidence intervals around the predictions, relative to a growing season with average conditions, are shown as dotted lines. 

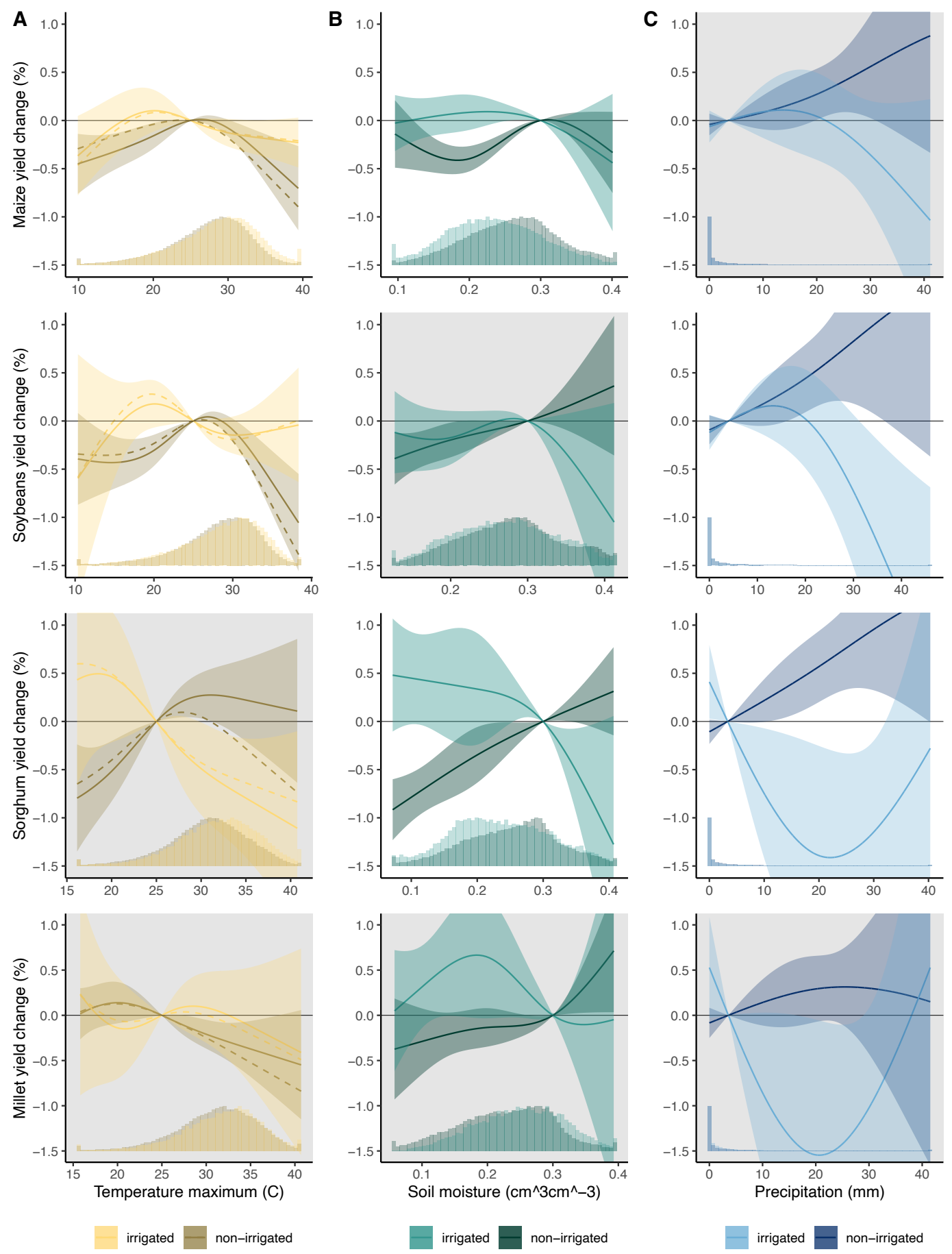

Extended Data Figure 6: Irrigation modifies the influences of climatic factors on crop yield and validates empirical separation of water supply and temperature stresses. Identical to Fig. 3 except that responses are estimated separately for each of the four crops rather than pooled. Grey backgrounds indicate models where either allowing for heterogeneity for irrigation does not improve performance (Extended Data Table 1) or where the response function is not significantly different from the null of zero effect at the $\mathrm{p}<0.10$ level. Outliers winsorized for display to their 0.5 and 99.5 percentiles for temperature and soil moisture and 1 and 99 percentiles for precipitation. Response functions plotted over the same range. 

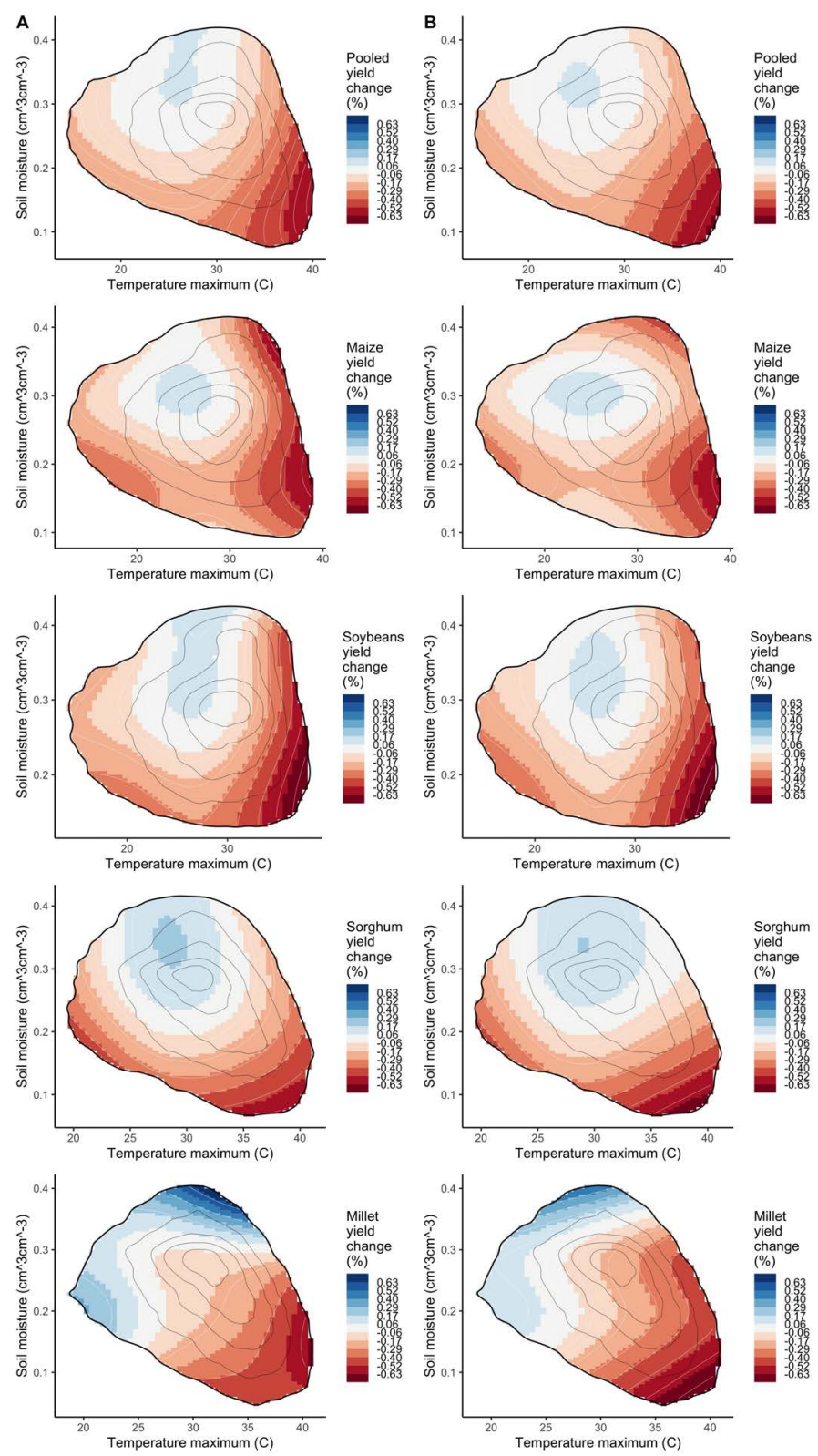

Extended Data Figure 7: Estimated influence of soil moisture and temperature on crop yield allowing for interaction effects. Response surfaces show the influence on yield of changing a day in the growing season from $25 \mathrm{C}$ and $0.2 \mathrm{~cm}^{3} \mathrm{~cm}^{-3}$ to a given temperature and soil moisture. (A) shows the estimated response surface, allowing the nonlinear influence of temperature and soil moisture to depend nonlinearly on the level of the other (Methods). (B) shows the estimated response surface allowing for nonlinear effects of temperature and soil moisture, but no interaction between the two (i.e. the same model as in Fig. 2). Black contours show the cropped-area-weighted distribution of daily growing season values across crops in the pooled surface and for each crop in their respective plots. Response functions are shown over the 95th percentile contour of the underlying distribution of daily values. White lines show contours of the estimated response surfaces to aid display. P-values for the null of no interaction effect are 0.738 for the pooled model, 0.295 for maize, 0.461 for soybeans, 0.956 for sorghum, and 0.141 for millet. 
A
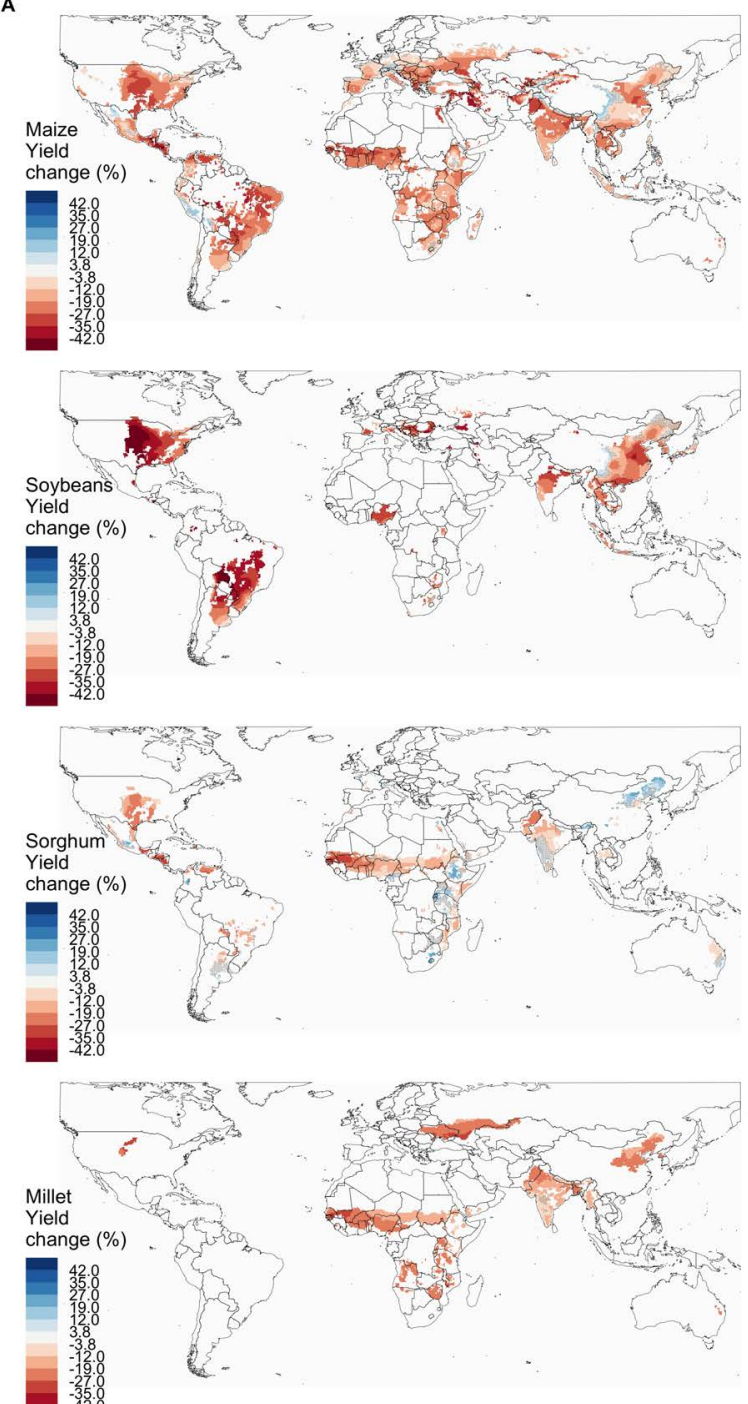

B
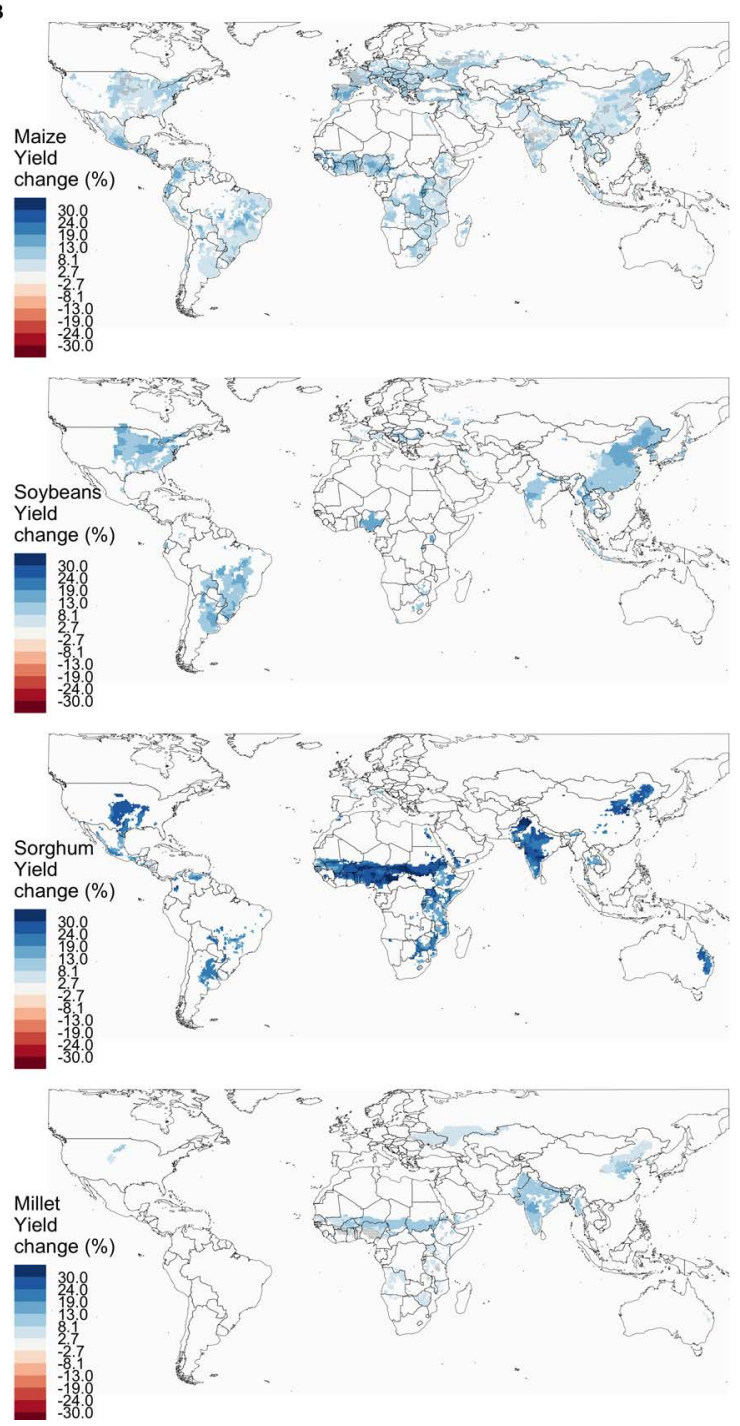

Extended Data Figure 8: Projected influence of climate change on maize, soybean, sorghum and millet yields. (A) Projected influence of climate change on crop yield using the TS model (SSP5-8.5, 2015-2035 to 2080-2100, averaged across six CMIP6 models, see Methods). (B) The difference in the projected influence of climate change on crop yield between the TS and TP models. Locations where five out of six of the models agree on the sign of effect are not stippled. Projections are made for locations where greater than 0.002 $\%$ (100 hectares) of the area is cultivated for a given crop. 
A
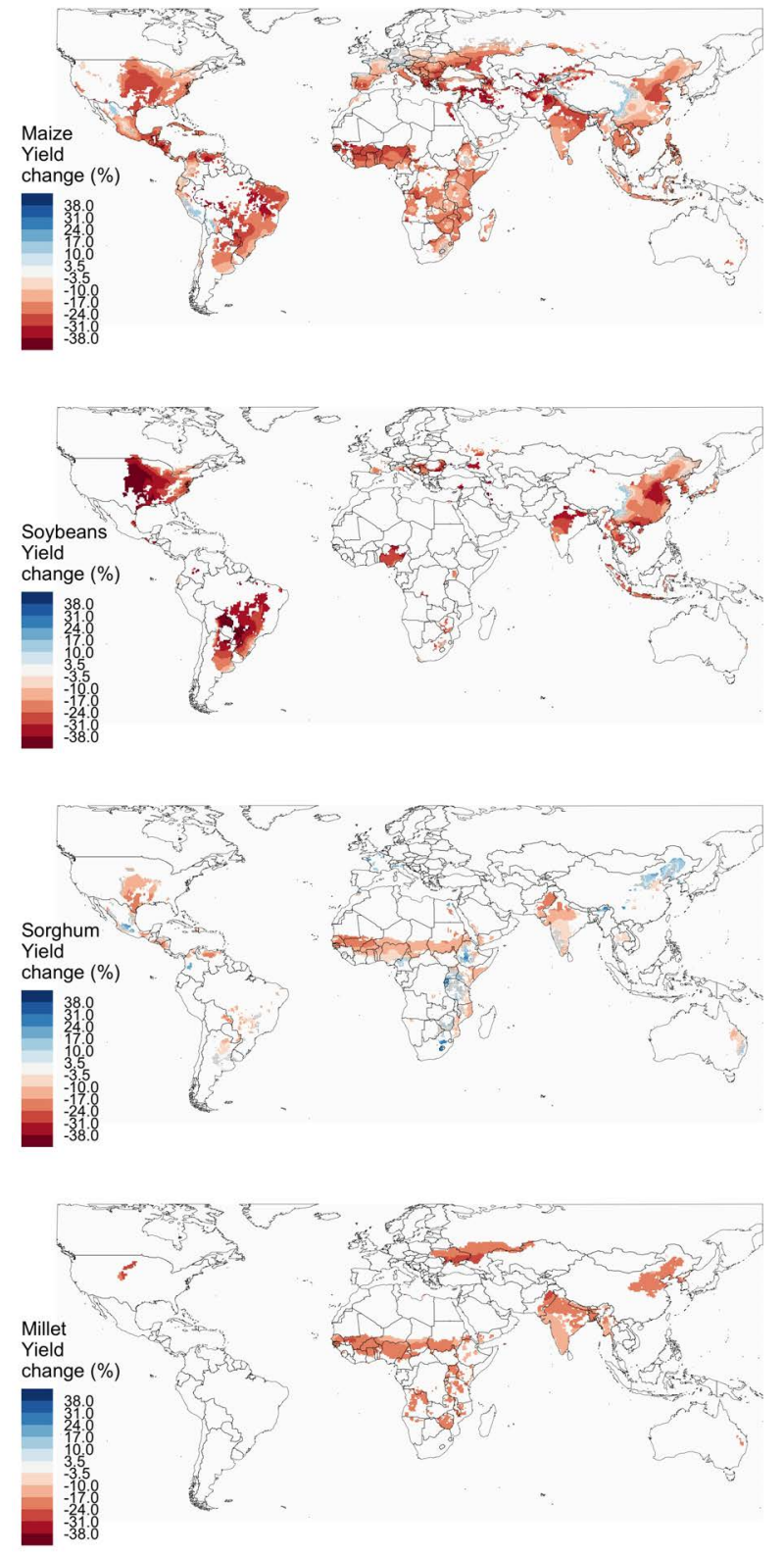

B
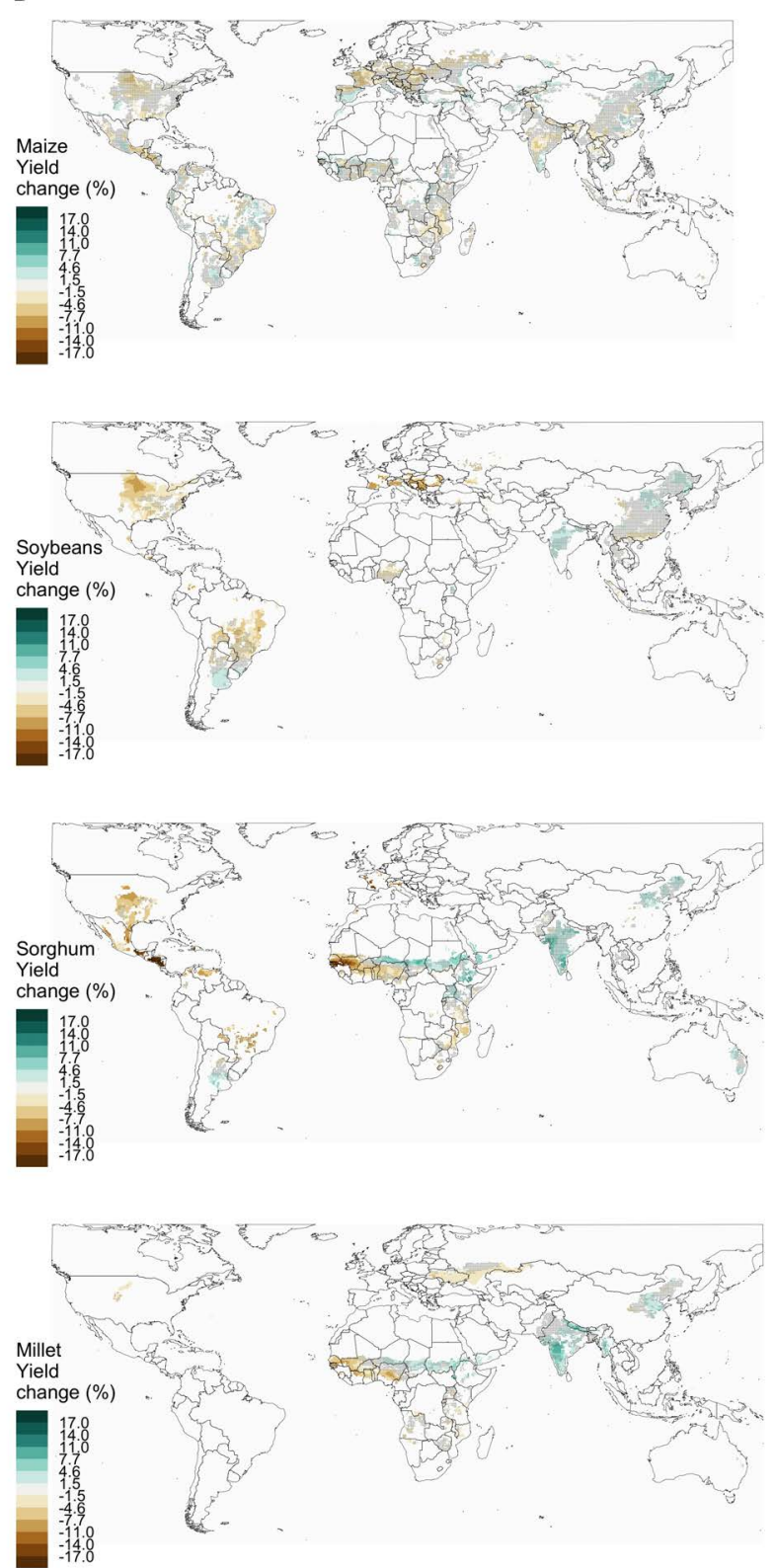

Extended Data Figure 9: Projected influence of climate change on maize, soybean, sorghum and millet yields due to temperature stress and soil moisture. (A) Projected influence of changes in temperature stress due to climate change on crop yield using the TS model (SSP5-8.5, 2015-2035 to 2080-2100, averaged across six CMIP6 models, see Methods). (B) Projected influence of changes in soil moisture due to climate change on crop yield using the TS model. Locations where five out of six of the models agree on the sign are not stippled. 

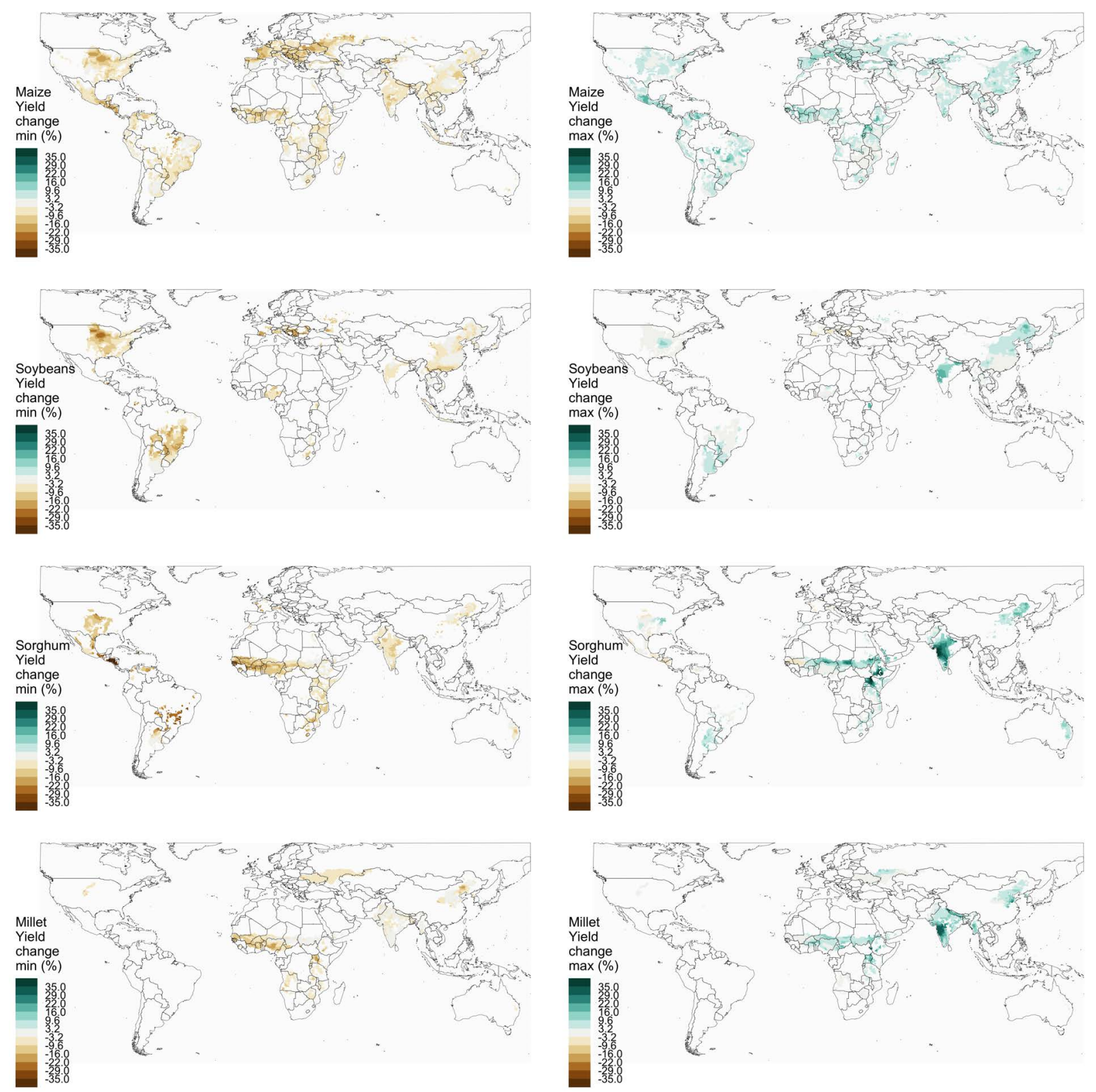

Extended Data Figure 10: CMIP6 model spread of the projected influence of changes in soil moisture on global crop yields. Maps show the pixel-wise minimum (left) and maximum (right) of projected soil moisture effects due to climate change across six CMIP6 models for maize, soybeans, sorghum and millet. 


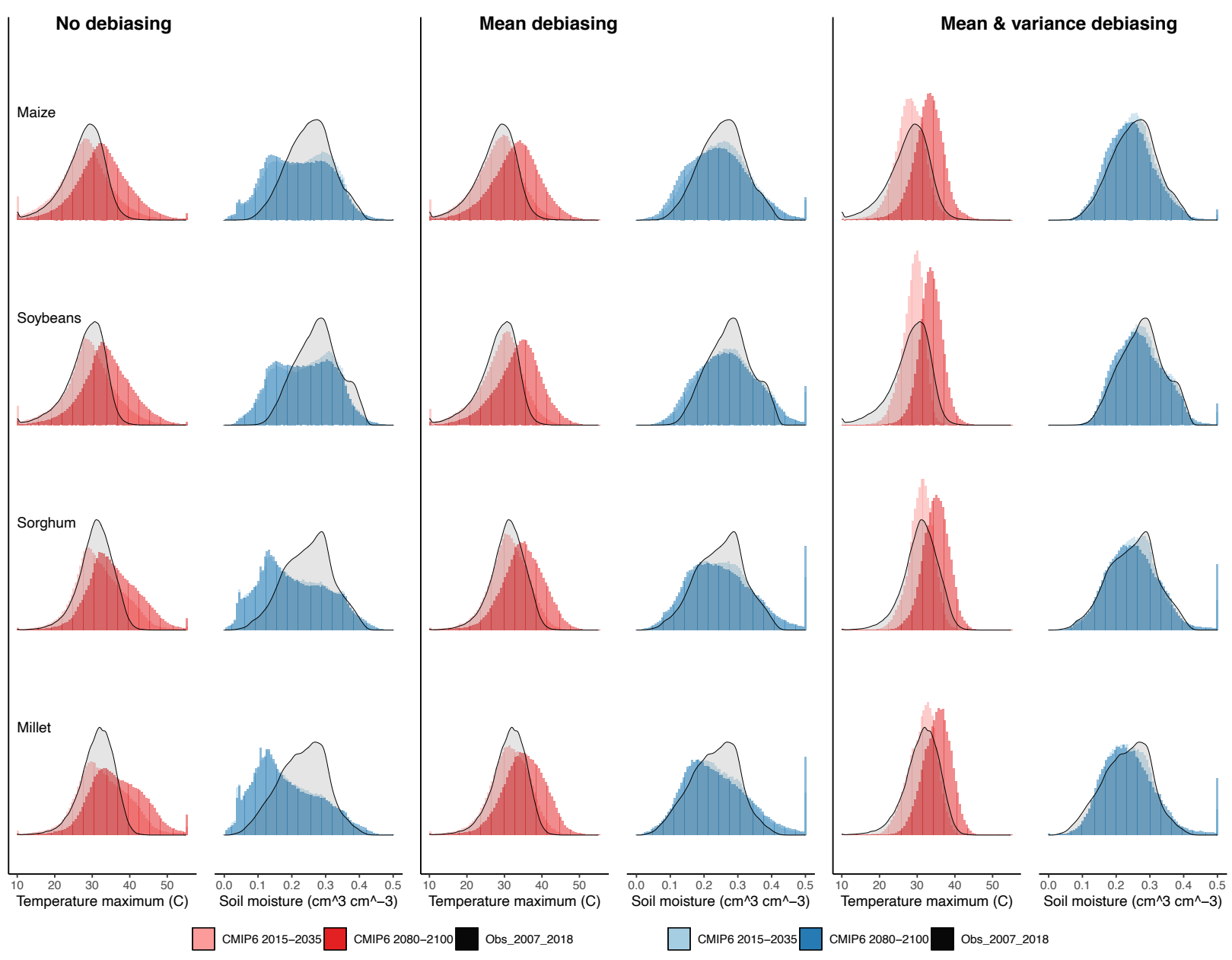

Extended Data Figure 11: Distribution of observed (black) and CMIP6 simulated (colored) daily growing season temperature and soil moisture over cropped areas before and after debiasing. Observations have been winsorized to $10^{\circ} \mathrm{C}$ and $55^{\circ} \mathrm{C}$ and to $0.5 \frac{\mathrm{cm}^{3}}{\mathrm{~cm}^{3}}$ for display. Light colors show near-future (2015-2035) values and dark colors show end of century (2080-2100) values. Middle and right columns show distributions after pixel-wise mean and mean and variance debiasing approaches were applied (Methods). 


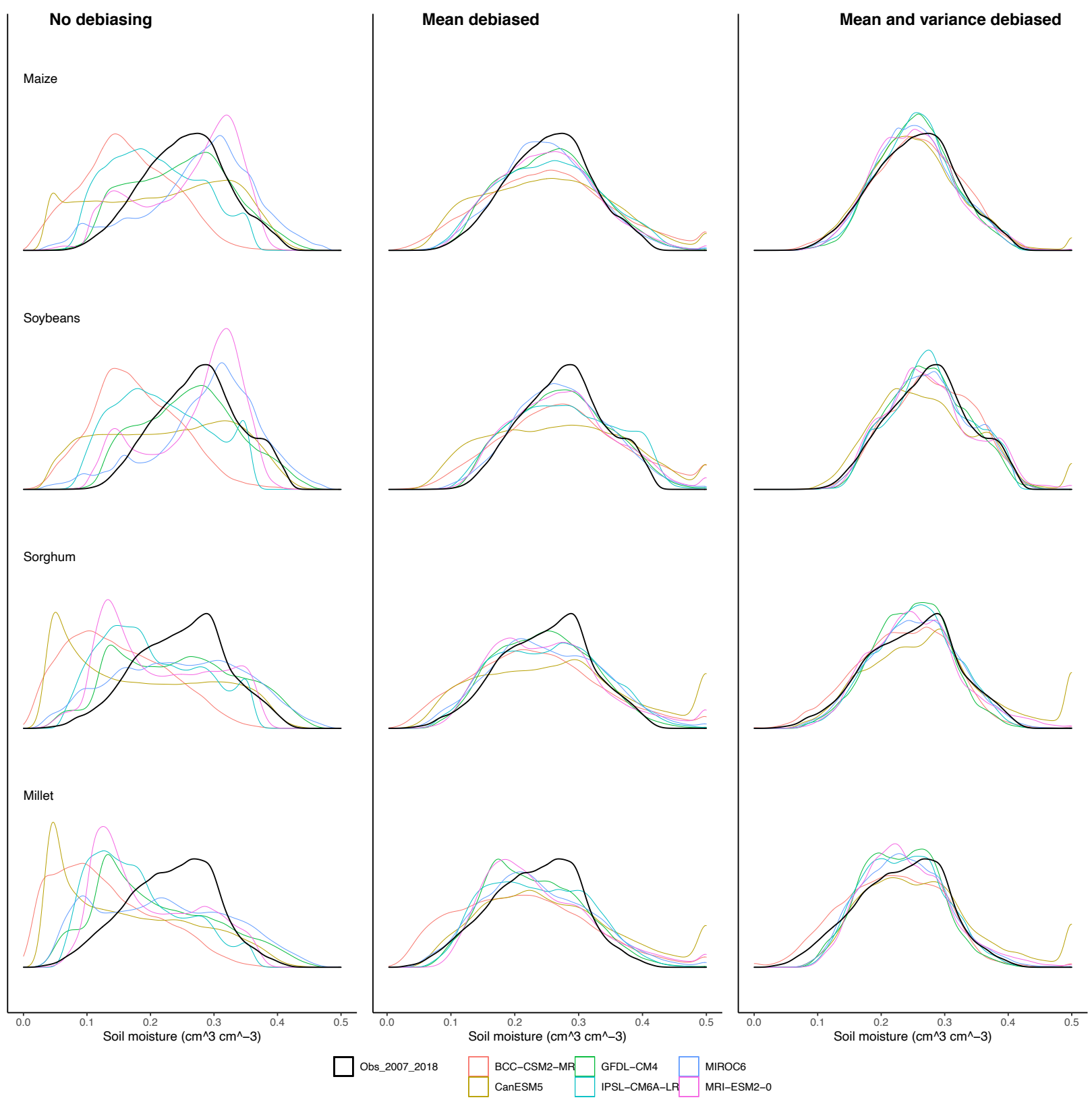

Extended Data Figure 12: Distribution of observed and CMIP6 simulated daily growing season soil moisture over cropped areas across CMIP6 models before and after debiasing. Observations have been winsorized to $10^{\circ} \mathrm{C}$ and $55^{\circ} \mathrm{C}$ and to $0.5 \frac{\mathrm{cm}^{3}}{\mathrm{~cm}^{3}}$ for display. CMIP6 data are near-future (2015-2035) values and observations are 2007-2018. Middle and right columns show distributions after pixel-wise mean and mean and variance debiasing approaches were applied (Methods). 


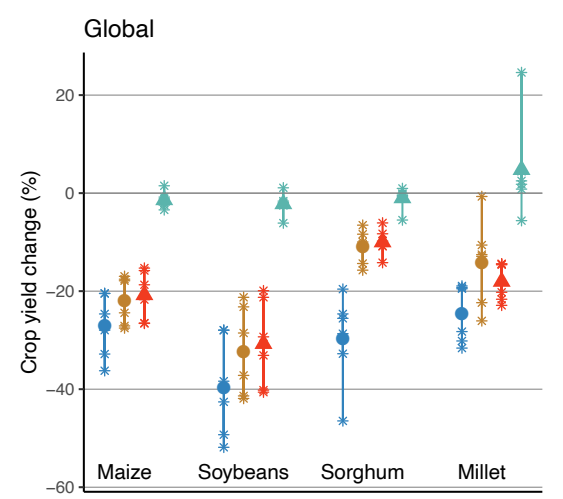

Central Europe

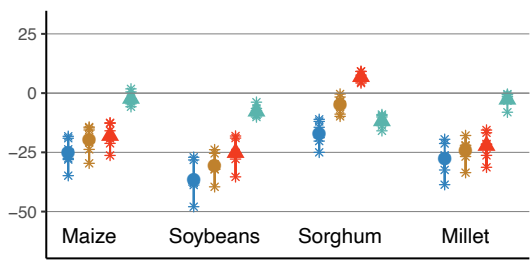

Central North America

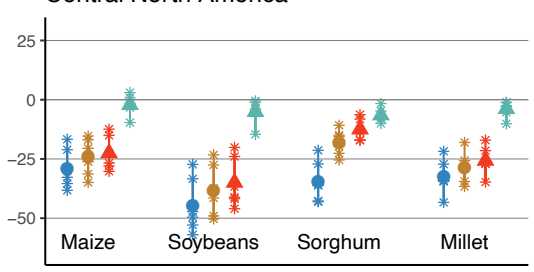

$$
\text { East Asia }
$$
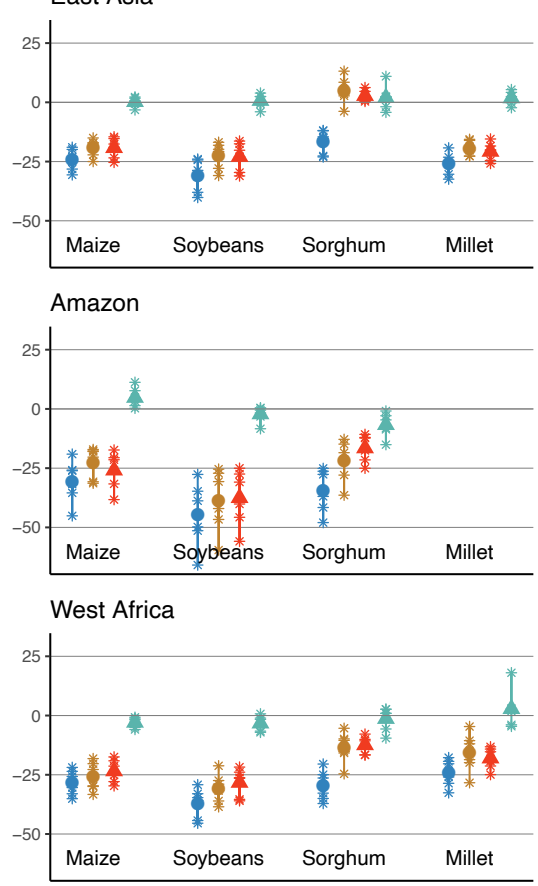
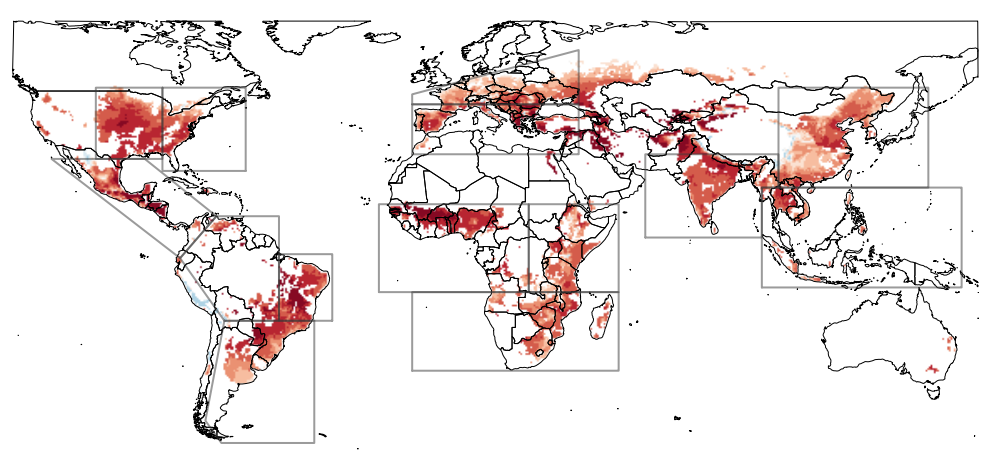

South Europe/Mediterranean

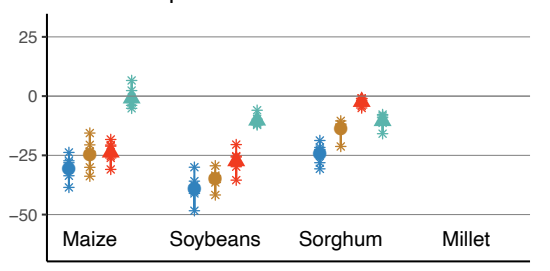

East North America

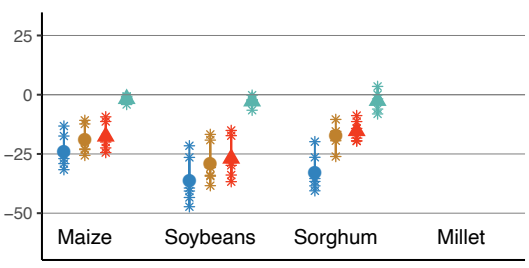

South Asia

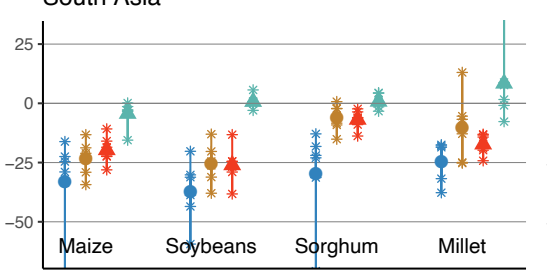

North-East Brazil
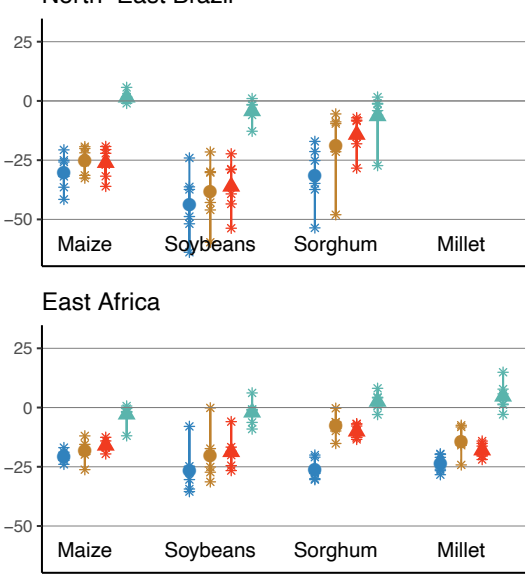

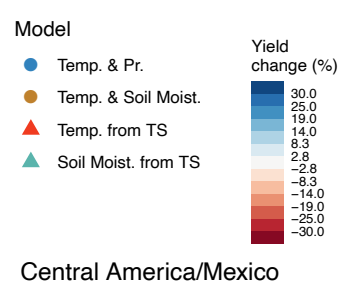

Central America/Mexico

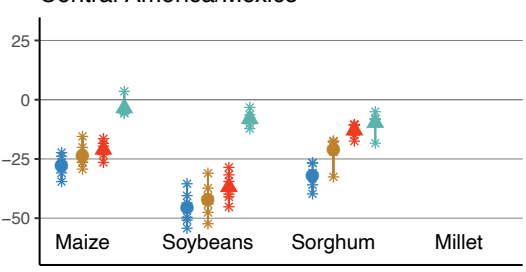

Southeast Asia

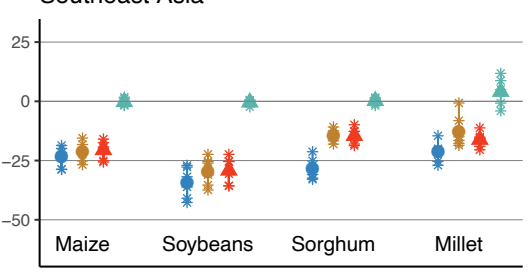

Southeastern South America
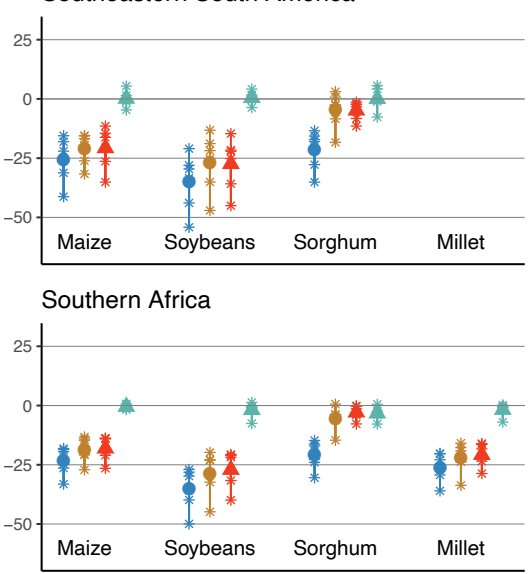

Extended Data Figure 13: Identical to Fig. 4 but using mean-debiased CMIP6 data. 

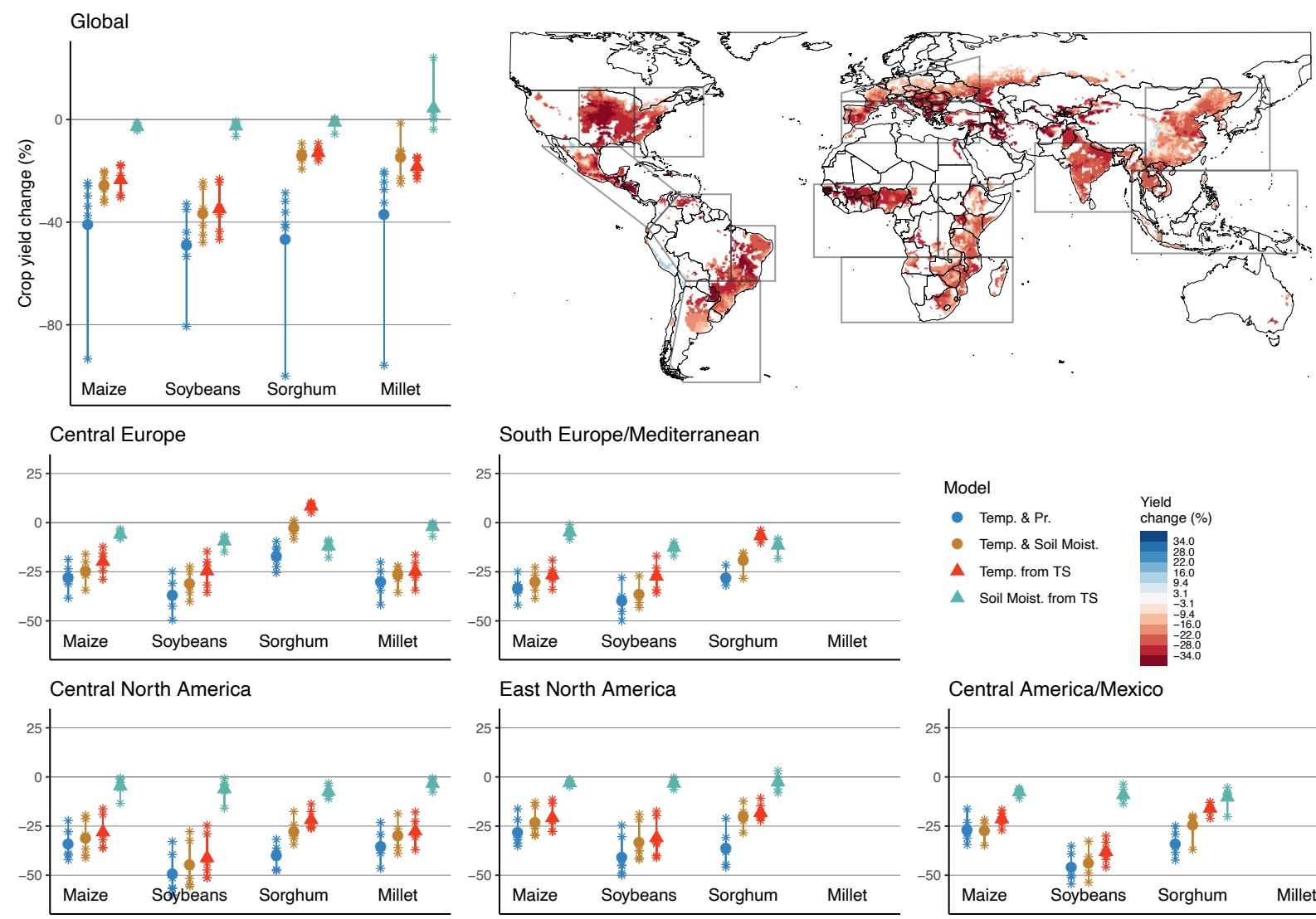

Central America/Mexico
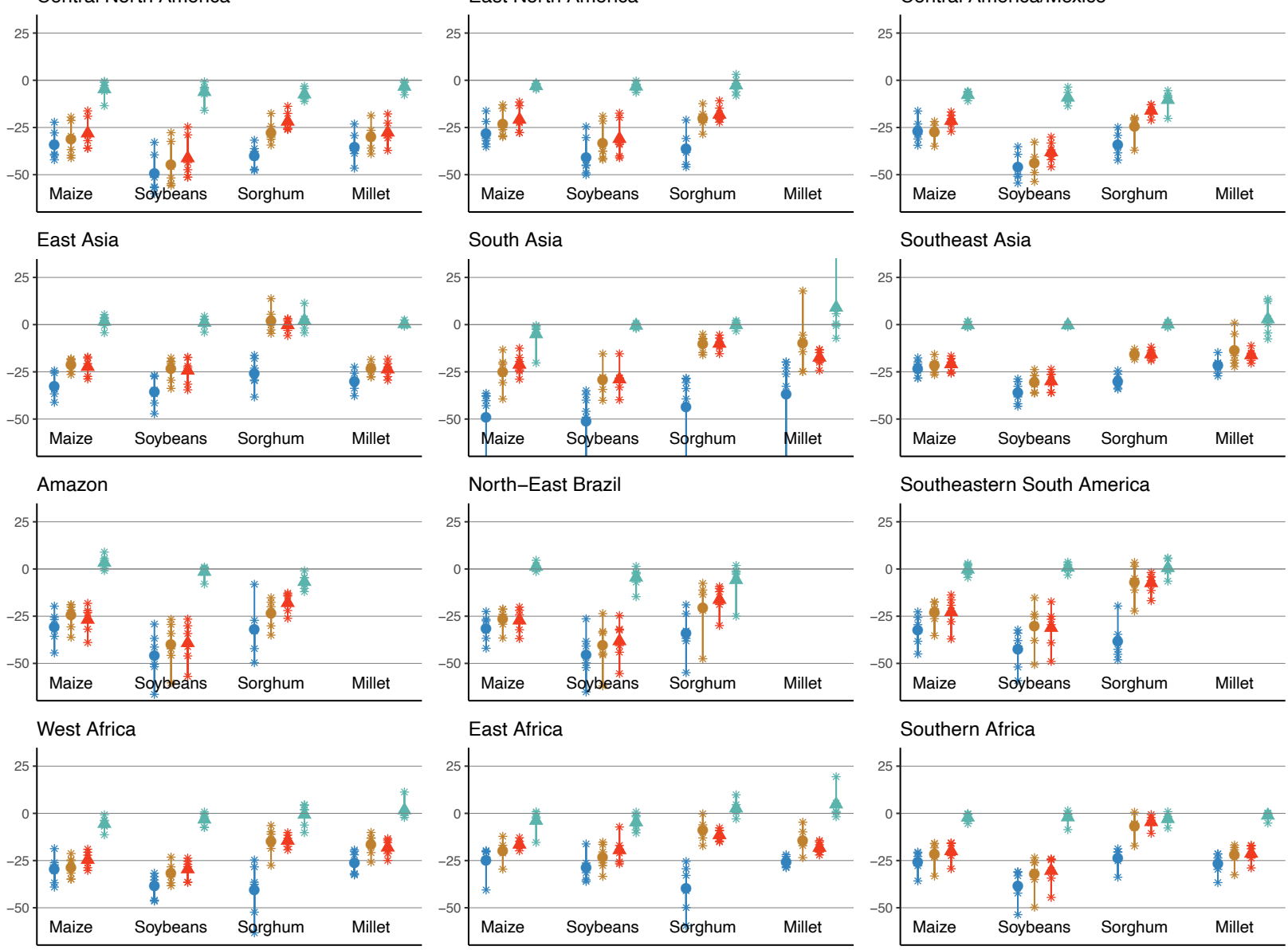

Extended Data Figure 14: Identical to Fig. 4 but using mean and variance debiased CMIP6 data. Note that the global mean values for one model are affected by a small number of pixels that have implausibly extreme precipitation values after variance-debiasing. 


\section{Supplementary Information}

\section{Supplementary Discussion 1: separating the influence of water supply and tem- perature stress in empirical crop models.}

Omitted variables bias: Here, we show analytically how accurately modelling water supply can empirically separate effects of water supply (i.e. soil moisture) from those of temperature stress (i.e. water demand and direct temperature effects). We also show how not fully accounting for water supply can lead to biased estimates of temperature stress. We do not attempt to separate effects due to changes in water demand and direct temperature effects because of the high colinearity between VPD and temperature and the low availability of global humidity measurements.

Assume that crop yield $y$, is determined by soil moisture, $m$, air temperature, $\tau$, and other unrelated factors $u$ according to:

$$
y=\beta_{0}+\beta_{\tau} \tau+\beta_{m} m+u
$$

and we wish to estimate the parameters $\beta_{\tau}$ and $\beta_{m}$ using observations. We assume that the error has mean zero and is uncorrelated with temperature and moisture.

If we estimate a model of yield as a function of temperature but not of moisture:

$$
y=b_{0}+b_{\tau} \tau+v
$$

we recover a biased estimate of the relationship between temperature and yields because part of the moisture effect is included in the estimated temperature effect: $b_{\tau}=\beta_{\tau}+\gamma_{\tau} \beta_{m}$, where $\gamma_{\tau}=\frac{\operatorname{cov}(m, \tau)}{\operatorname{var}(\tau)} \cdot \gamma_{\tau}$ can also be interpreted as the coefficient from a regression of $m$ on $\tau$ - i.e.:

$$
m=\gamma_{0}+\gamma_{\tau} \tau+\mu
$$

This is a classic case of omitted variables bias where the estimated influence of temperature is biased because soil moisture is omitted from the model, but is correlated with both yield and temperature. If we assume, consistent with observations, that temperature and soil moisture are negatively correlated $\left(\gamma_{\tau}<0\right)$ and that soil moisture has a positive influence on yield $\left(\beta_{m}>0\right)$, then the bias $\left(\gamma_{\tau} \beta_{m}\right)$ will be negative, meaning that a model estimating the effect of temperature on yield not controlling for moisture would over-estimate damages of temperature by conflating hot and dry or cool and wet conditions.

If instead we estimate a model controlling for an imperfect proxy for moisture, such as precipitation, $p$ :

$$
y=\alpha_{0}+\alpha_{\tau p} \tau+\alpha_{p} p+\phi
$$

where

$$
m=\theta_{0}+\theta_{p} p+\theta_{\tau} \tau+r
$$

and the error term $r$ is uncorrelated with $p$, then we again get a biased estimate of the influence of temperature on yield: $\alpha_{\tau p}=\beta_{\tau}+\theta_{\tau} \beta_{m}$. If $p$ is a good proxy for $m$, 
then the relationship between temperature and soil moisture is weaker after conditioning on precipitation than before, i.e. $\left|\theta_{\tau}\right|<\left|\gamma_{\tau}\right|$, so adding $p$ into the model as a proxy for $m$ reduces bias because $\left|\theta_{\tau} \beta_{m}\right|<\left|\gamma_{\tau} \beta_{m}\right|$. This is the case in our empirical setting. The better proxy we use for $m$, the smaller the bias is. If we directly measure $m$ and include it in the regression we, of course, recover an unbiased estimate of $\beta_{\tau}$ and $\beta_{m}$. Though no measure will ever perfectly capture root zone soil moisture across the globe, we demonstrate through this analysis that available soil moisture measurements provide a better proxy for crop water supply than do available measurements of precipitation.

The intuition from this linear example extends to the setting where yield is a nonlinear function of temperature and moisture, which we demonstrate through simulation, and which is discussed further in the material which this derivation is based on: Wooldridge, 2002, "Econometric Analysis of Cross Section and Panel Data", Chapter 4 [15].

Simulations: Simulations match the analytical derivations above (Extended Data Fig. 1). In this simulation, temperature, soil moisture and precipitation are drawn from a joint normal distribution with co-variances chosen to match those observed in the historical data (Extended Data Fig. 1A). Daily yield growth is modeled as a function of daily temperature and soil moisture. Annual yields are calculated as the product of daily yield growth, following [1] and [52], such that log yields are the sum of daily yield growth. The effects of temperature and moisture on yield are then estimated using the same regression approach as in the empirical yield model (Eqn. 1).

We find in the simulation, consistent with the analytical derivation above and the empirical results, that the estimated temperature response is negatively biased relative to the true response when the response of yield to temperature is estimated without any controls (Extended Data Fig. 1B). Controlling for precipitation reduces this bias slightly. Controlling for soil moisture recovers the prescribed temperature effect.

Simulations prescribing the influence of temperature to be quadratic while keeping the effect of soil moisture linear show that the unconditional temperature response and the temperature response conditional on precipitation are equally biased across the support (Extended Data Fig. 1C). Note that a constant bias of the marginal effect of temperature will generate a larger and larger level difference the greater the change in temperature. Controlling for soil moisture again recovers the prescribed temperature effect.

In our empirical analysis, Fig. 2, we find that the temperature response in the TP models is exaggerated relative to that of the TS models, especially when temperatures are high. We recover this magnified bias at hot temperatures in the TP model relative to the TS model in simulation by prescribing a nonlinear influence of soil moisture on yield that is consistent with the estimated soil moisture response function (Fig. 2, Extended Data Fig. 1D). Specifically, we prescribe soil moisture to benefit yield up to a point, after which it has no effect. This magnifies bias at high temperatures relative to low temperatures because, as shown above, bias in the influence of temperature is the product of the partial influence of soil moisture on yield and the correlation between soil moisture and temperature. Thus, since soil moisture has a stronger influence on yields at lower soil moisture, and lower soil moisture is correlated with hotter conditions, this leads to a larger bias in the TP model relative to the TS model at higher temperatures. And since soil moisture has a weak (or even 
negative) influence on yields at higher soil moisture, and higher soil moisture is correlated with lower temperatures, this leads to a smaller bias in the TP model relative to the TS model at lower temperatures.

We also show in simulation that the stronger association between temperature and soil moisture at hot temperatures relative to cold temperatures (Extended Data Fig. 2C) can also lead to greater bias in the estimated influence of temperature on yield in the TP model relative to the TS model at hot temperatures (Extended Data Fig. 1E,F). These simulations suggest that the observed increase in bias in the estimated temperature response in the TP model relative to the TS model at hot temperatures is likely due to a combination of both a stronger relationship between temperature and soil moisture at higher temperatures and a stronger influence of soil moisture on yields at low soil moistures, which tend to occur in hot conditions.

Supplementary Discussion 2: Alternate cross-validation approaches. In the main text we describe how TS models have improved performance relative to TP models and how individual $\mathrm{T}$ and $\mathrm{S}$ models show relatively equal performance. Here we also note that modelling the nonlinear effect of daily temperature rather than seasonal temperature improves performance substantially ( $\mathrm{R}^{2}$ more than doubles) for maize, soybeans and sorghum, while for millet there is little difference. This could be due to either nonlinear daily effects of temperature on water supply, water demand, or temperature stress. Modelling the effect of soil moisture at the daily or seasonal resolution has little influence on the model performance, likely due to the linearity of the soil moisture effect over a large portion of its support.

In this section we conduct two additional cross-validation tests. First, in Supplementary Information Table 1 we conduct a bootstrap approach where within each of the crossvalidation folds we bootstrap 500 samples from the training set and use that to make predictions in the validation set. We then calculate the mean and standard deviation of $\mathrm{R}^{2}$ for the 500 predictions of the held out data. Second, in Supplementary Information Table 2 we repeat the cross-validation experiment in Extended Data Table 1, but split training and testing datasets by country to test the ability of the model to spatially extrapolate.

We find that the patterns in Extended Data Table 1 are largely replicated in these two experiments; the two minor exceptions are discussed below. First, while the TS model has a higher $\mathrm{R}^{2}$ in the primary and bootstrapped cross-validation experiments for all crops, in the spatial cross-validation experiment the $\mathrm{R}^{2}$ of the TP model is 0.001 higher for millet. Second, and related, the individual performance of soil moisture relative to that of temperature is lower for millet in the spatial cross-validation experiment than in the other two experiments. These two difference could either be due to noise, given the small size of differences relative to the standard deviations of the $\mathrm{R}^{2}$ s calculated in Supplementary Information Table 1, or potentially due to heterogeneity in the effect of soil moisture on millet yields across countries. 


\begin{tabular}{|c|c|c|c|c|c|c|c|c|c|}
\hline & Crop & Model & Resolution & $\mathrm{T}$ & $\mathrm{P}$ & $\mathrm{S}$ & $\mathrm{TP}$ & TS & TSP \\
\hline 1 & Maize & quadratic & day & 0.055 & 0.019 & 0.052 & 0.058 & 0.068 & 0.065 \\
\hline 2 & & & & $(0.004)$ & $(0.003)$ & $(0.004)$ & $(0.004)$ & $(0.006)$ & $(0.006)$ \\
\hline 3 & Maize & quadratic & season & 0.019 & 0.026 & 0.054 & 0.063 & 0.071 & 0.071 \\
\hline 4 & & & & $(0.003)$ & $(0.003)$ & $(0.004)$ & $(0.004)$ & $(0.006)$ & $(0.006)$ \\
\hline 5 & Maize & cubic spline & day & 0.059 & 0.018 & 0.064 & 0.059 & 0.088 & 0.084 \\
\hline 6 & & & & $(0.004)$ & $(0.003)$ & $(0.004)$ & $(0.005)$ & $(0.006)$ & $(0.006)$ \\
\hline 7 & Maize & cubic spline & season & 0.017 & 0.023 & 0.063 & 0.062 & 0.085 & 0.08 \\
\hline 8 & & & & $(0.004)$ & $(0.003)$ & $(0.003)$ & $(0.005)$ & $(0.006)$ & $(0.007)$ \\
\hline 9 & Maize & irrigation & day & 0.087 & & 0.097 & 0.058 & 0.096 & \\
\hline 10 & & & & $(0.007)$ & & $(0.007)$ & $(0.007)$ & $(0.007)$ & \\
\hline 11 & Maize & interaction & day & & & & & 0.078 & \\
\hline 12 & & & & & & & & $(0.009)$ & \\
\hline 13 & Soybeans & quadratic & day & 0.092 & 0.031 & 0.089 & 0.086 & 0.115 & 0.102 \\
\hline 14 & & & & $(0.006)$ & $(0.007)$ & $(0.007)$ & $(0.00$ & $(0.00$ & $(0.011)$ \\
\hline 15 & Soybeans & quadratic & season & 0.043 & 0.056 & 0.089 & 0.103 & 0.116 & 0.11 \\
\hline 16 & & & & $(0.005)$ & $(0.006)$ & $(0.007)$ & $(0.009)$ & $(0.008)$ & $(0.011)$ \\
\hline 17 & Soybeans & cubic spline & day & 0.098 & 0.031 & 0.083 & 0.088 & 0.118 & 0.101 \\
\hline 18 & & & & (0.007) & $(0.007)$ & (0.009) & $(0.01)$ & $(0.01)$ & $(0.013)$ \\
\hline 19 & Soybeans & cubic spline & season & 0.037 & 0.05 & 0.082 & 0.108 & 0.117 & 0.111 \\
\hline 20 & & & & $(0.008)$ & $(0.006)$ & (0.009) & $(0.01)$ & $(0.011)$ & $(0.013)$ \\
\hline 21 & Soybeans & irrigation & day & 0.126 & & 0.119 & 0.107 & 0.124 & \\
\hline 22 & & & & $(0.011)$ & & $(0.012)$ & $(0.012)$ & $(0.01$ & \\
\hline 23 & Soybeans & interaction & day & & & & & 0.101 & \\
\hline 24 & & & & & & & & $(0.013)$ & \\
\hline 25 & Sorghum & quadratic & day & 0.01 & 0.007 & 0.037 & 0.012 & 0.036 & 0.032 \\
\hline 26 & & & & $(0.008)$ & $(0.002)$ & $(0.006)$ & (0.008) & $(0.009)$ & $(0.009)$ \\
\hline 27 & Sorghum & quadratic & season & -0.009 & 0.013 & 0.036 & 0.017 & 0.036 & 0.032 \\
\hline 28 & & & & $(0.009)$ & $(0.003)$ & $(0.006)$ & $(0.008)$ & $(0.009)$ & $(0.009)$ \\
\hline 29 & Sorghum & cubic spline & day & 0.01 & 0.004 & 0.032 & 0.01 & 0.0 & 0.025 \\
\hline 30 & & & & $(0.008)$ & $(0.003)$ & $(0.007)$ & $(0.00$ & $(0.011)$ & $(0.011)$ \\
\hline 31 & Sorghum & cubic spline & season & -0.015 & 0.011 & 0.031 & 0.016 & 0.031 & 0.022 \\
\hline 32 & & & & $(0.009)$ & $(0.004)$ & $(0.007)$ & $(0.009)$ & $(0.011)$ & $(0.012)$ \\
\hline 33 & Sorghum & irrigation & day & 0.016 & & 0.038 & 0.001 & 0.031 & \\
\hline 34 & & & & $(0.013)$ & & (0.011) & $(0.011)$ & $(0.013)$ & \\
\hline 35 & Sorghum & interaction & day & & & & & -0.011 & \\
\hline 36 & & & & & & & & (0.018) & \\
\hline 37 & Millet & quadratic & day & 0.026 & 0.002 & 0.014 & 0.022 & 0.018 & 0.015 \\
\hline 38 & & & & $(0.005)$ & $(0.003)$ & (0.008) & $(0.005)$ & (0.009) & $(0.009)$ \\
\hline 39 & Millet & quadratic & season & 0.02 & 0.002 & 0.015 & 0.02 & 0.02 & 0.016 \\
\hline 40 & & & & $(0.005)$ & $(0.004)$ & $(0.007)$ & (0.006) & (0.008) & $(0.008)$ \\
\hline 41 & Millet & cubic spline & day & 0.025 & -0.001 & 0.016 & 0.016 & 0.02 & 0.011 \\
\hline 42 & & & & $(0.005)$ & $(0.004)$ & (0.008) & $(0.007)$ & $(0.009)$ & $(0.01)$ \\
\hline 43 & Millet & cubic spline & season & 0.024 & -0.003 & 0.008 & 0.015 & 0.012 & 0.005 \\
\hline 44 & & & & $(0.006)$ & $(0.007)$ & $(0.009)$ & $(0.008)$ & $(0.01)$ & $(0.011)$ \\
\hline 45 & Millet & irrigation & day & 0.013 & & 0.005 & 0.008 & -0.002 & \\
\hline 46 & & & & $(0.01)$ & & $(0.014)$ & $(0.01)$ & $(0.015)$ & \\
\hline 47 & Millet & interaction & day & & & & & -0.007 & \\
\hline 48 & & & & & & & & $(0.015)$ & \\
\hline
\end{tabular}

Supplementary Information Table 1: Boostrapped cross-validated model within $\mathbf{R}^{2} \mathbf{s}$. Same as Extended Data Table 1 except that within each of the cross-validation folds we bootstrap 500 samples from the training set and use that to make predictions in the validation set. We then calculate the mean and standard deviation, in parentheses, of within $\mathrm{R}^{2}$ for the 500 predictions of the held out data. 


\begin{tabular}{|c|c|c|c|c|c|c|c|c|c|}
\hline & Crop & Model & Resolution & $\mathrm{T}$ & $\mathrm{P}$ & $\mathrm{S}$ & $\mathrm{TP}$ & $\mathrm{TS}$ & TSP \\
\hline 1 & Maize & quadratic & day & 0.057 & 0.016 & 0.047 & 0.059 & 0.066 & 0.062 \\
\hline 2 & Maize & quadratic & season & 0.024 & 0.026 & 0.052 & 0.065 & 0.072 & 0.071 \\
\hline 3 & Maize & cubic spline & day & 0.061 & 0.015 & 0.057 & 0.061 & 0.085 & 0.080 \\
\hline 4 & Maize & cubic spline & season & 0.022 & 0.022 & 0.057 & 0.063 & 0.084 & 0.078 \\
\hline 5 & Maize & irrigation & day & 0.081 & & 0.091 & 0.055 & 0.089 & \\
\hline 6 & Maize & interaction & day & & & & & 0.079 & \\
\hline 7 & Soybeans & quadratic & day & 0.087 & 0.031 & 0.084 & 0.089 & 0.111 & 0.106 \\
\hline 8 & Soybeans & quadratic & season & 0.042 & 0.054 & 0.080 & 0.104 & 0.108 & 0.110 \\
\hline 9 & Soybeans & cubic spline & day & 0.096 & 0.026 & 0.079 & 0.090 & 0.117 & 0.103 \\
\hline 10 & Soybeans & cubic spline & season & 0.042 & 0.045 & 0.076 & 0.109 & 0.113 & 0.113 \\
\hline 11 & Soybeans & irrigation & day & 0.127 & & 0.110 & 0.108 & 0.121 & \\
\hline 12 & Soybeans & interaction & day & & & & & 0.108 & \\
\hline 13 & Sorghum & quadratic & day & 0.012 & 0.005 & 0.041 & 0.012 & 0.041 & 0.038 \\
\hline 14 & Sorghum & quadratic & season & -0.002 & 0.010 & 0.038 & 0.017 & 0.039 & 0.035 \\
\hline 15 & Sorghum & cubic spline & day & 0.013 & 0.005 & 0.035 & 0.013 & 0.036 & 0.032 \\
\hline 16 & Sorghum & cubic spline & season & -0.006 & 0.008 & 0.036 & 0.015 & 0.040 & 0.030 \\
\hline 17 & Sorghum & irrigation & day & 0.027 & & 0.043 & 0.011 & 0.043 & \\
\hline 18 & Sorghum & interaction & day & & & & & -0.005 & \\
\hline 19 & Millet & quadratic & day & 0.030 & -0.0001 & 0.012 & 0.024 & 0.019 & 0.014 \\
\hline 20 & Millet & quadratic & season & 0.025 & -0.0003 & 0.013 & 0.022 & 0.021 & 0.017 \\
\hline 21 & Millet & cubic spline & day & 0.028 & -0.004 & 0.010 & 0.017 & 0.016 & 0.005 \\
\hline 22 & Millet & cubic spline & season & 0.026 & -0.006 & 0.005 & 0.014 & 0.010 & -0.001 \\
\hline 23 & Millet & irrigation & day & 0.009 & & 0.013 & 0.014 & 0.004 & \\
\hline 24 & Millet & interaction & day & & & & & 0.016 & \\
\hline
\end{tabular}

Supplementary Information Table 2: Model $\mathbf{R}^{2} \mathbf{s}$ cross-validated with across country folds. Same as Extended Data Table 1 except that during cross-validation, blocks of data from countries are randomly partitioned into 10 folds, rather than observations being randomly partitioned. Training is done on 9 of the folds and models are evaluated on the remaining fold. 


\section{References}

[1] Schlenker, W. \& Roberts, M. J. Nonlinear temperature effects indicate severe damages to U.S. crop yields under climate change. Proceedings of the National Academy of Sciences 106, 15594-15598 (2009).

[2] Mbow, C. et al. Food Security. Climate Change and Land: an IPCC special report on climate change, desertification, land degradation, sustainable land management, food security, and greenhouse gas fluxes in terrestrial ecosystems (2021).

[3] Schlenker, W. \& Lobell, D. B. Robust negative impacts of climate change on African agriculture. Environmental Research Letters 5 (2010).

[4] Lobell, D. B., Schlenker, W. \& Costa-Roberts, J. Climate trends and global crop production since 1980. Science 333, 616-620 (2011).

[5] Ortiz-Bobea, A., Ault, T. R., Carrillo, C. M., Chambers, R. G. \& Lobell, D. B. Anthropogenic climate change has slowed global agricultural productivity growth. Nature Climate Change 11, 306-312 (2021).

[6] Proctor, J., Hsiang, S., Burney, J., Burke, M. \& Schlenker, W. Estimating global agricultural effects of geoengineering using volcanic eruptions. Nature 560, 480-483 (2018).

[7] Vogel, E. et al. The effects of climate extremes on global agricultural yields. Environmental Research Letters 14 (2019).

[8] Ortiz-Bobea, A., Wang, H., Carrillo, C. M. \& Ault, T. R. Unpacking the climatic drivers of US agricultural yields. Environmental Research Letters 14 (2019).

[9] Rigden, A. J., Mueller, N. D., Holbrook, N. M., Pillai, N. \& Huybers, P. Combined influence of soil moisture and atmospheric evaporative demand is important for accurately predicting US maize yields. Nature Food 1, 127-133 (2020).

[10] Chaves, M. M. et al. How plants cope with water stress in the field. Photosynthesis and growth. Annals of Botany 89, 907-916 (2002).

[11] Buckley, T. N. How do stomata respond to water status? New Phytologist 224, 21-36 (2019).

[12] Siega, T. D. C., Bertoldo, E. \& Vismara, L. D. S. Cavitation and embolism in plants: literature review. Australian Journal of Basic and Applied Sciences 10-14 (2018).

[13] Waqas, M. A. et al. Thermal stresses in maize: Effects and management strategies. Plants 10, 1-23 (2021).

[14] Schauberger, B. et al. Consistent negative response of US crops to high temperatures in observations and crop models. Nature Communications 8 (2017). 
[15] Wooldridge, J. M. Econometric Analysis of Cross Section and Panel Data (MIT Press, Cambridge, MA, 2002).

[16] Zeppetello, L. R., Tetreault-Pinard, E., Battisti, D. S. \& Baker, M. B. Identifying the sources of continental summertime temperature variance using a diagnostic model of land-atmosphere interactions. Journal of Climate 33, 3547-3564 (2020).

[17] Agnolucci, P. et al. Impacts of rising temperatures and farm management practices on global yields of 18 crops. Nature Food 1, 562-571 (2020).

[18] Seneviratne, S. I. et al. Investigating soil moisture-climate interactions in a changing climate: A review. Earth-Science Reviews 99, 125-161 (2010).

[19] Lobell, D. B. et al. The critical role of extreme heat for maize production in the United States. Nature Climate Change 3, 497-501 (2013).

[20] Mueller, N. D. et al. Cooling of US Midwest summer temperature extremes from cropland intensification. Nature Climate Change 6, 317-322 (2015).

[21] Zhu, P. \& Burney, J. Untangling irrigation effects on maize water and heat stress alleviation using satellite data. Hydrology and Earth System Sciences Discussions 1-30 (2021).

[22] Carter, E. K., Melkonian, J., Riha, S. J. \& Shaw, S. B. Separating heat stress from moisture stress: Analyzing yield response to high temperature in irrigated maize. Environmental Research Letters 11 (2016).

[23] Novick, K. A. et al. The increasing importance of atmospheric demand for ecosystem water and carbon fluxes. Nature Climate Change 6, 1023-1027 (2016).

[24] Liu, L. et al. Soil moisture dominates dryness stress on ecosystem production globally. Nature Communications 11, 1-9 (2020).

[25] Wijewardana, C. et al. Quantifying soil moisture deficit effects on soybean yield and yield component distribution patterns. Irrigation Science 36, 241-255 (2018).

[26] Dorigo, W. et al. ESA CCI Soil Moisture for improved Earth system understanding: State-of-the art and future directions. Remote Sensing of Environment 203, 185-215 (2017).

[27] Gruber, A., Scanlon, T., Van Der Schalie, R., Wagner, W. \& Dorigo, W. Evolution of the ESA CCI Soil Moisture climate data records and their underlying merging methodology. Earth System Science Data 11, 717-739 (2019).

[28] CPC Global Unified Gauge-Based Analysis of Daily Precipitation. URL https://psl.noaa.gov/data/gridded/data.cpc.globalprecip.html.

[29] FAOSTAT Crops and Livestock Products. URL https://www.fao.org/faostat. 
[30] Hsiang, S. M. Climate Econometrics. Annual Review of Resource Economics 1-33 (2016).

[31] Eyring, V. et al. Overview of the Coupled Model Intercomparison Project Phase 6 (CMIP6) experimental design and organization. Geoscientific Model Development 9, 1937-1958 (2016).

[32] Agnolucci, P. et al. Impacts of rising temperatures and farm management practices on global yields of 18 crops. Nature Food 1, 562-571 (2020).

[33] Sánchez, B., Rasmussen, A. \& Porter, J. R. Temperatures and the growth and development of maize and rice: A review. Global Change Biology 20, 408-417 (2014).

[34] Hirasawa, T. \& Hsiao, T. C. Some characteristics of reduced leaf photosynthesis at midday in maize growing in the field. Field Crops Research 62, 53-62 (1999).

[35] Troy, T. J., Kipgen, C. \& Pal, I. The impact of climate extremes and irrigation on US crop yields. Environmental Research Letters 10, 054013 (2015).

[36] Ashraf, M. \& Habib-ur-Rehman. Interactive effects of nitrate and long-term waterlogging on growth, water relations, and gaseous exchange properties of maize (Zea mays L.). Plant Science 144, 35-43 (1999).

[37] Li, Y., Guan, K., Schnitkey, G. D., DeLucia, E. \& Peng, B. Excessive rainfall leads to maize yield loss of a comparable magnitude to extreme drought in the United States. Global Change Biology 25, 2325-2337 (2019).

[38] Rosenzweig, C., Tubiello, F. N., Goldberg, R., Mills, E. \& Bloomfield, J. Increased crop damage in the US from excess precipitation under climate change. Global Environmental Change 12, 197-202 (2002).

[39] Voesenek, L. A. \& Bailey-Serres, J. Flood adaptive traits and processes: An overview. New Phytologist 206, 57-73 (2015).

[40] Jägermeyr, J. et al. Climate impacts on global agriculture emerge earlier in new generation of climate and crop models. Nature Food (2021).

[41] Berg, A., Sheffield, J. \& Milly, P. C. Divergent surface and total soil moisture projections under global warming (2017).

[42] Cicchino, M., Rattalino Edreira, J. I. \& Otegui, M. E. Heat stress during late vegetative growth of maize: Effects on phenology and assessment of optimum temperature. Crop Science 50, 1432-1436 (2010).

[43] Lorenz, D. J., Nieto-Lugilde, D., Blois, J. L., Fitzpatrick, M. C. \& Williams, J. W. Downscaled and debiased climate simulations for North America from 21,000 years ago to 2100AD. Scientific Data 3, 1-19 (2016).

[44] Proctor, J. Atmospheric opacity has a nonlinear effect on global crop yields. Nature Food 2, 166-173 (2021). 
[45] Taylor, C. A. \& Schlenker, W. Environmental Drivers of Agricultural Productivity Growth: CO2 Fertilization of US Field Crops. National Bureau of Economic Research Working Paper Series No. 29320 (2021).

[46] Ford, T. W., Harris, E. \& Quiring, S. M. Estimating root zone soil moisture using nearsurface observations from SMOS. Hydrology and Earth System Sciences 18, 139-154 (2014).

[47] CPC Global Daily Temperature. URL https://psl.noaa.gov/data/gridded/data.cpc.globaltem]

[48] Monfreda, C., Ramankutty, N. \& Foley, J. A. Farming the planet: 2. Geographic distribution of crop areas, yields, physiological types, and net primary production in the year 2000. Global Biogeochemical Cycles 22, 1-19 (2008).

[49] Sacks, W. J., Deryng, D., Foley, J. A. \& Ramankutty, N. Crop planting dates: An analysis of global patterns. Global Ecology and Biogeography 19, 607-620 (2010).

[50] Algorithm Theoretical Baseline Document (ATBD) Supporting Product Version 06.1. Tech. Rep., Earth Observation Data Centre for Water Resources Monitoring (EODC) GmbH (2021).

[51] Stefan, S., Verena, H., Karen, F. \& Burke, J. Global Map of Irrigation Areas version 5. Food and Agriculture, AQUASTAT (2013).

[52] Blackman, V. H. The Compound Interest Law and Plant Growth. Annals of Botany XXXIII, 353-360 (1919). 